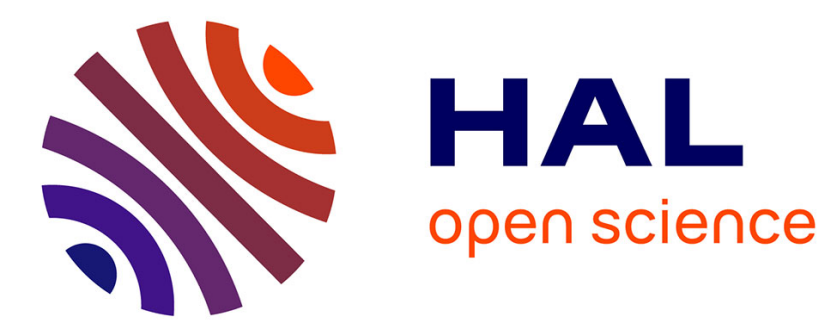

\title{
Molecular Simulation of Solid-Solid Phase Transitions
} Patrice Bordat, Alain Marbeuf, Ross Brown

\section{To cite this version:}

Patrice Bordat, Alain Marbeuf, Ross Brown. Molecular Simulation of Solid-Solid Phase Transitions. Molecular Simulation, 2007, 32 (12-13), pp.971-984. 10.1080/08927020600863022 . hal-00514993

\section{HAL Id: hal-00514993 https://hal.science/hal-00514993}

Submitted on 4 Sep 2010

HAL is a multi-disciplinary open access archive for the deposit and dissemination of scientific research documents, whether they are published or not. The documents may come from teaching and research institutions in France or abroad, or from public or private research centers.
L'archive ouverte pluridisciplinaire HAL, est destinée au dépôt et à la diffusion de documents scientifiques de niveau recherche, publiés ou non, émanant des établissements d'enseignement et de recherche français ou étrangers, des laboratoires publics ou privés. 


\section{Molecular Simulation}

Journal of

Experimental Nanoscience

Taylor \& Francis

Taglor \& Francis Group

\section{Molecular Simulation of Solid-Solid Phase Transitions}

\begin{tabular}{|c|c|}
\hline Journal: & Molecular Simulation/Journal of Experimental Nanoscience \\
\hline Manuscript ID: & GMOS-2006-0072 \\
\hline Journal: & Molecular Simulation \\
\hline $\begin{array}{r}\text { Date Submitted by the } \\
\text { Author: }\end{array}$ & 30-Apr-2006 \\
\hline Complete List of Authors: & $\begin{array}{l}\text { Bordat, Patrice; Ictpcm } \\
\text { Marbeuf, Alain; cpmoh } \\
\text { Brown, Ross; LCTPCM }\end{array}$ \\
\hline Keywords: & phase transition, dl_poly, alkane, p-terphenyl, aluminophosphate \\
\hline
\end{tabular}

\section{SCHOLARONE \\ Manuscripts}




\author{
Patrice Bordat ${ }^{+}$, Alain Marbeuf ${ }^{++}$and Ross Brown ${ }^{+*}$ \\ + Laboratoire de chimie théorique et de physico-chimie moléculaire, \\ umr $5624 \mathrm{du}$ CNRS et de l'Université de Pau et des pays de l'Adour, \\ IFR, rue J. Ferry, 64075 PAU Cedex, France \\ ${ }^{++}$Centre de physique moléculaire optique et hertzienne \\ umr 5098 du CNRS et de l'Université de Bordeaux I, \\ 33405 TALENCE Cedex, France
}

April 30, 2006

\begin{abstract}
Applications of DL_POLY to solid-solid phase transitions are reviewed, with particular attention to how details of the mechanisms of the transitions may be extracted from molecular dynamics simulations. Two examples in molecular crystals are discussed: the order-disorder transition of $p$-terphenyl initiated at around $200 \mathrm{~K}$ by the unlocking of ring flipping; and the rotator phases of $n$-alkanes with around 20 carbon atoms per chain, showing distinct molecular mechanisms in the dynamics just below the melting points of odd and even chains. Covalent-ionic materials are represented by an application to an aluminophophate molecular sieve, $\mathrm{AlPO}_{4^{-}}-5$.
\end{abstract}

\title{
1 Introduction
}

Solid-solid phase transitions are perhaps among the most interesting aspects of the dynamics of the solid state. On the one hand it is fascinating that one and the same molecule can exist in different packings, held together by the balance of cohesive forces, anisotropy of molecular shapes and interactions and thermal agitation. On the other, solid-solid transitions are

\footnotetext{
${ }^{* *}$ Corresponding author. Email: ross.brown@univ-pau.fr
} 
of practical importance as in ferroelectric materials and polymorphism of pharmaceutical compounds.

Transitions between phases with well defined structures can be investigated by estimation of the free energies. For example, a zero temperature approximation in which enthalpies, based on the electronic energies of optimised structures, are computed by periodic ab initio methods, is particularly useful for pressure induced transitions of covalent materials $[1,2]$. Another method is to perform a sequence of molecular dynamics simulations of a finite but representative piece of the material at different values of the control parameter, usually the temperature or the pressure, and to wait for a spontaneous transition in the system. Although progress in ab initio molecular dynamics has been fast over the last couple of decades, the size of system that can be simulated is usually too small for a direct application of the second method. This method, which is often more informative about the mechanisms of transitions, such as growing disorder, the rôle of local fluctuations, or the growth of correlations, is therefore mostly applied within the framework of molecular dynamics with empirical potentials. There should not therefore be strong electronic reorganisation during the transition. Molecular solids are thus obvious applications, but examples in covalent materials do exist, mainly in disorder or displacive transitions such as the $\alpha-\beta$ transition of cristobalite[3]. Occasionally an empirical model may adequately mimic recoordination of atoms in covalent materials, as we have found for the transition from $\mathrm{AlPO}_{4}-53(\mathrm{~B})$ to $\mathrm{AlPO}_{4}-53(\mathrm{C})[4]$. Although direct simulation is intuitively appealing, one should be aware that the transition will depend to some extent on the system size and on the time one can afford to simulate. Too short a simulation may lead one to miss a sluggish transition for values of the control parameter close to the true value for an infinite system.

The advantages of direct observation of the transition in the molecular dynamics simulations will be illustrated below by reviewing three examples, two molecular solids and an aluminophosphate molecular sieve. DL_POLY is particularly convenient for this kind of work due to the ability to accommodate a very wide range of empirical potential functions, including freezing atoms or groups of atoms or treatment of parts of the system as rigid units. Use of tabulated potentials is also possible, allowing for example for patching the sometimes catastrophic potential well present at zero separation in 6-exp potentials. Another advantage of the code is the possibility of iso-stress simulations in a general (triclinic) simulation box, allowing for observation of spontaneous changes of the crystal space group.

The general procedure illustrated below is to first set up a model of the ordered phase, based on experimental data from X-ray or neutron scattering, and to minimise the total 
Page 3 of 39 potential energy in a succession of NVT, NpT and N $\sigma \mathrm{T}$ simulations coupled to a cold heat bath (Berendsen thermo- and barostats in our case[5]). This precaution is necessary since even small differences between the experimental structure and the equilibrium structure of the model may lead to high contact forces and instability of the model. The simulation may then be equilibrated at a state point close to the transition. Some order parameter will change during the transition, which will proceed between states with differing space groups, the group of the ordered phase being a sub-group of the disordered phase. Usually, one has to be content with driving the models of molecular solids from the ordered to the less ordered phase, for example by increasing the temperature stepwise through the transition in a set of thermostated simulations, since orientational relaxation times close to the transition may be very long and prevent observation of the reverse transition from disorder to order. An exception is the aluminophosphate molecular sieve simulated below, which transited reversibly between the high and the low temperature phases.

\section{The order-disorder transition of $p$-terphenyl.}

[Figure 1 about here.]

Our first example is $p$-terphenyl, figure 1, a widely studied molecular solid with an order-disorder transition, an archetype for anti-ferroelastic disorder-transitions. The linear polyphenyls are an interesting class of compounds from the point of view of solid-solid phase transitions. Biphenyl, $p$-terphenyl and $p$-quaterphenyl crystals are monoclinic at room temperature, with two molecules per unit cell. Individual molecules are flat on average, but show unusually large thermal motions about their long axes, corresponding to superposition of the expected libration about the long axis and of flipping of the phenyl rings through the mean molecular plane. The equilibrium inter-ring torsion of $p$-terphenyl in the gas phase, close to $45^{\circ}[6]$, is a compromise between $\pi$ conjugation and repulsion between the orthohydrogens. Ring flipping is still present in the solid at room temperature, but the molecule is flattened by the crystal pressure, with torsions in the range $15-30^{\circ}$. Modelling these interesting systems is particularly challenging because of the strong coupling of internal and external degrees of freedom.

The phase transition is associated with the locking of the ring flipping motion at lower temperatures. See reference [7] for a complete overview on the transition. Measurements at $110 \mathrm{~K}$ revealed a doubling of the unit cell along the $\vec{a}$ and $\vec{b}$ directions, leading to a triclinic phase with four inequivalent molecules per cell[8], distinguished mainly by their tilts off the $\vec{b}$ 
axis and their equilibrium inter-ring torsions, which range from $15^{\circ}$ to $27^{\circ}$. The central ring is tilted alternately above and below the plane of the outer rings for adjacent molecules along the $\vec{a}$ and $\vec{b}$ directions, see figure 1 . The phase transition actually occurs at $193 \mathrm{~K}$, as revealed by differential scanning calorimetry[9]. The 'individual molecule' dynamics of ring flipping was investigated by quasi-elastic incoherent neutron scattering and proton NMR[10, 11, 12]. The results at high temperature indicate uncorrelated flipping of individual molecules over a (torsional) barrier of apparent height $\approx 1 \mathrm{kcal} / \mathrm{mol}$. Pre-transitional growth and slowing down of intermolecular correlations were also deduced from these experimental studies. Cell angles $\alpha$ and $\gamma$ were indistinguishable from $90^{\circ}$ so that it is convenient to adopt a pseudomonoclinic cell (figure 1) containing 8 molecules.

$P$-terphenyl was modelled with an empirical potential developped specifically for representing the low temperature phase and the transition. The main difficulty being is to obtain accurate ring twists in the low temperature phase. We first adopted standard intermolecular potentials from the literature, but found that they did not adequately describe the low temperature phase. We therefore adapted them according to a scheme proposed by Yokoi and Nishikawa[13], in which non-bonded 6-exp terms depend on the partial charges of the atoms. Partial charges were found to be important for the precise packing though unimportant in the total potential energy. Finally, the inter-ring torsional potential was tuned to provide a satisfactory compromise representation of the low and the high temperature phases, potential II in reference [7]. In this review, we prefer to dwell on qualitative features of the transition, full numerical details being available in the published papers[14, 7, 15] - Numerical procedures within DL_POLY were standard and will not be reproduced here, except for a comment on two points. With hindsight, and based on our subsequent experience with the $n$-alkanes, we note in passing that we would today prefer larger models than the $2 \times 2 \times 2$ pseudo-monoclinic cells (64 molecules) used at the time (though quite challenging with the then available computer power). The second point is that we have not yet found any significantly different behaviour in models of molecular crystals integrated with either Ewald summation or the shifted potential approximation for the electrostatic forces. The electrostatic contribution to the total energy is small, since partial charges are of the order of $\pm 0.1 e$ and the overall interactions often are quadrupole-quadrupole or higher order. Use of neutral charge groups would be equally effective (see also Wolf et al.[16] for a perspective on this problem).

Analysis of atomic motions deduced from diffraction data led to the belief that in the high temperature phase, the molecules librate about their long axes and the central ring flips 
Page 5 of 39 back and forth through the plane of the outer rings[17]. Ring flipping can be characterised by 5 angles: the inter-ring torsions $\phi_{1}$ and $\phi_{2}$, and the twist of the rings out of the mean molecular plane, $\theta_{1}$ and $\theta_{3}$ for the outer rings and $\theta_{2}$ for the central ring, in the notation of Baudour[17]. As a first illustration of the kind of information that can be extracted from molecular simulation of solid-solid transitions, figure 2(a) shows a scatter diagram of the instantaneous values of the inter-ring torsions $\phi_{2}$ vs. $\phi_{1}$ at $300 \mathrm{~K}$, above the transition temperature.

$\phi_{1}$ and $\phi_{2}$ are equal and opposite in sign, showing that in the solid the molecule is esentially centro-symmetric and never adopts the cork-screw conformation found in the gas phase. In Baudour's analysis, the libration of the molecule about the long axis was decomposed into three modes in the twists $\theta$ in an effective single molecule potential: an external mode $\theta_{1}=\theta_{2}=\theta_{3}$ (libration), a $g$ internal mode $\theta_{1}=\theta_{3}=-\theta_{2} / 2$ and a $u$ internal mode $\theta_{1}=\theta_{2}=\theta_{3}$. Figure $2(\mathrm{~b})$ shows a scatter plot of $\theta_{1}$ against $\theta_{2}$. We observe that the points are clustered around the line $\theta_{1}=-\theta_{2} / 2$ as predicted. The average twists of the central ring out of the mean plane, $14.5^{\circ}$ and $14.1^{\circ}$ at $180 \mathrm{~K}$ and $300 \mathrm{~K}$ are close to $2 / 3$ of the mean torsions, $20.2^{\circ}$ and $17.3^{\circ}$, as expected, and close to the experimental values[18], $13.2^{\circ}$ and $13.4^{\circ}$. Figures 2(c) and (d) compare the simulated distributions of $\theta_{1}$ at $180 \mathrm{~K}$ and $300 \mathrm{~K}$, with the distributions deduced by Baudour et al.[18] from a simple decomposition of the experimental librational tensors into a well-hopping contribution between the above mean values and overall librations. Again agreement with experiment is good. Finally, figure 2(e), we use the distribution of $\theta_{2}, P\left(\theta_{2}\right)$ to deduce an effective 'single particle' potential for ring flipping, analagous to a potential of mean force, by defining $V\left(\theta_{2}\right)$ such that

$$
P(\theta) \propto \exp \left(-V(\theta) / k_{B} T\right)
$$

This has been done in $2(\mathrm{e})$ at two temperatures, $180 \mathrm{~K}$ and $300 \mathrm{~K}$. The motion is quite anharmonic, so that the effective potential barrier for ring flipping is temperature dependent: about $1.5 \mathrm{kcal} / \mathrm{mol}$ at $180 \mathrm{~K}$ and $1.1 \mathrm{kcal} / \mathrm{mol}$ at $300 \mathrm{~K}$, in agreement with the activation energy deduced from quasi-elastic incoherent neutron scattering and NMR data[12].

[Figure 2 about here.]

The high temperature phase of $p$-terphenyl is monoclinic with two molecules per unit cell, which are flat on average. The low tempreature, triclinic phase contains four molecules distinguished by the values of the locked inter-ring torsions. The values of these torsions averaged over all equivalent molecules in the simulation are monitored at standard pressure 
in figure $3(\mathrm{a})$, as the temperature is increased by stages throught the transition. We observe that the four average values are constant at low temperature, but drop sharply and merge to a common, much smaller value between 180 and $200 \mathrm{~K}$ which brackets the transition temperature of the model. Above this temperature, molecules are flat on average. The same study can be done at a higher pressure, $2.5 \mathrm{kbar}$ in figure $3(\mathrm{~b})$. Torsions at low temperature are smaller than at standard pressure due to the flattening influence of the crystal pressure. Correspondingly, the transition temperature is lowered, to arround $150 \mathrm{~K}$ since the crystal pressure has brought individual molecules closer to the top of the barrier in the 'single molecule' torsional potential in figure 2(e). The simulated transition points are in fair agreement with experiment, see figure $3(d)$.

[Figure 3 about here.]

A current extension of this work is the dynamics of domain walls in the low temperature phase. Considering a row of molecules in either the $a$ or $b$ crystal directions in the high temperature phase, the central phenyl ring will be flipping chaotically to either side of the mean molecular planes, which are all oriented parallel in a given row (see figure 1). As the crystal is cooled through the transition, flipping slows down and becomes correlated among neighbouring molecules, until eventually the motion stops and the rings lock alternately "above" and "below" the mean plane, as shown in the figure. This transition occurs spontaneously and without coordination in different parts of the material, so that four types of energetically equivalent domains appear in the low temperature phase, related either by symmetry elements lost at the transition (e.g glide planes parallel to $b c$ ) in which case a ferroelastic domain boundary occurs at their interface; or by a half period translational mismatch, leading to an anti-phase boundary[19], see figure 4.

[Figure 4 about here.]

Ferroelastic domain walls are expected from group theoretical considerations to be flat and immobile, and may be revealed by polarised microscopy[19]. Anti-phase walls on the other hand are invisible and may be mobile and sinuous. Since less is therefore known about anti-phase boundaries than about ferroelastic domain walls and because the evidence is indirect, we set up molecular models of anti-phase walls parallel to $b c$ and examined their dynamics. Larger models were used than previously: 18x8x4=576 molecules, with half the $b c$ sheets along $a$ in one domain and the other half in the other, see figure 5. Molecules in the sheets (blue in figure 5) were frozen to prevent interference between the domains 
Page 7 of 39 while allowing periodic boundary conditions to continue to apply in DL_POLY in the $b$ and $c$ directions.

[Figure 5 about here.]

Molecules at the anti-phase boundary must have frustrated interactions with one or other domain, see figure 4, and hence may be expected to fluctuate between the left and the right domain. Motion of the domain wall is monitored by recording whether the ring position of a given molecule is in accordance with what would fit with the packing in its initial domain, or with the opposite domain, or whether the molecule is close to flat (strained environment). These types of molecules are coloured green, red and grey in figure 6. The initially flat wall, one molecule thick, turns out to be quite mobile, even at low temperatures.

[Figure 6 about here.]

Indeed, as shown in figure 6 , after only $80 \mathrm{ps}$ of dynamics at $50 \mathrm{~K}$, the wall has thickened to to a stable value of about $5 b c$ planes deep and has started to diffuse away from its initial position. From a practical point of view, figures such as figure 6 may be conveniently prepared with script-driven molecular graphics programs, in this case Rasmol[20]. The MD trajectory is post-processed to obtain the ring torsions and an XYZ file is written with normal, out of order and flat molecules grouped together. It is then straightforward to generate a script for plotting the molecules with different colours. Our simulations[21] show that the domain walls are mobile even at low temperatures, which is consistent with indirect evidence from frequency jumps of the absorption spectra of probe molecules in the material[22]. They are easily deformed and may be pinned by impurity molecules, tetracene in this case, again in agreement with experiment[19].

\section{Rotator phases in $n$-alkanes}

The properties of $n$-alkanes, $\mathrm{C}_{n} \mathrm{H}_{2 n+2}$, abbreviated here to $\mathrm{C} n$, with chain lengths in the range $n=10-30$ carbon atoms exhibit an interesting odd-even effect. The layered crystal packing of all the $n$-alkanes is highly anisotropic, with molecules densely packed side by side to form sheets. But whereas the even $n$-alkanes with $n$ from 8 to 20 have triclinic unit cells with one molecule per unit cell, tilted off the layer normal, the odd alkanes from $n=13-21$ are orthorhombic $(Z=4)$, with molecules arranged perpendicular to the layers, see figure 7 .

[Figure 7 about here.] 
The alternation of properties extends to the dynamics. Thus, the melting points, for example, increase overall with chain length, but alternate between adjacent members of the series. Even more surprising is that while the even members of the series melt directly from the ordered phase to the isotropic liquid, the odd members pass through one or more disordered "rotator" phases before attaining the liquid state. Properties of the odd and even members of the series converge as the chain length reaches about 30 carbon atoms. Not surprisingly, this variety of behaviour has attracted widespread interest, with varied techniques, including X-ray([23]) and neutron scattering[24], positron lifetime spectroscopy[25], IR[26] and Raman[27] spectroscopy, NMR[28] and pulsed field gradient NMR[29], differential scanning calorimetry[30] and pVT measurements[31] (see reference [32] for more details).

The layered structure and ranslational symmetry are maintained in the rotator phases, but molecules several orientations, separated by $90^{\circ}\left(R_{\mathrm{I}}\right.$ phase $)$ or $60^{\circ}\left(R_{\mathrm{II}}\right)$ jumps about the long axis. Combination of simulations and neuton scattering led to a coherent picture of motions in the rotator phases of the odd alkanes [33, 34, 35, 24]. However, most earlier simulations of the rotator phases dealt with their properties at equilibrium, often with model systems prepared in disordered configurations. Less is known, particularly at the molecular level, about the mechanisms of the transitions from the ordered phase to the rotator phases and why they are absent in the even alkanes, but present in odd chains of comparable length. Indeed we are not aware of earlier simulations of the even alkanes and therefore performed a homogeneous set of molecular dynamics simulations of odd and even alkanes[32]: octadecane, $\mathrm{C}_{18} \mathrm{H}_{38}$, nonadecane, $\mathrm{C}_{19} \mathrm{H}_{40}$, eicosane, $\mathrm{C}_{20} \mathrm{H}_{42}$ and docosane, $\mathrm{C}_{22} \mathrm{H}_{46}$ (abbreviated below as C18, C19, C20 and C22). Simulations in the isothermal, iso-stress ensemble allowed observation of individual molecular motions during spontaneous transitions to the rotator phase or the liquid. They showed how a molecule may start to turn in the initially unfavourable packing of the ordered phase. We used an adapted form of the COMPASS force field as proposed by Sun[36], with intramolecular bond-bond, bond-angle and other cross terms dropped, since they could not be accomodated in DL_POLY. All molecular dynamics simulations were performed with dl_poly 2.13[37], in the Berendsen isothermal and iso-stress ensemble (N $\sigma \mathrm{T})$, with timestep $0.5 \mathrm{fs}$. Simulation boxes of 600 molecules $(\mathrm{Z}=1 ; 10 \mathrm{x} 10 \mathrm{x} 6$ cells, except C19: $\mathrm{Z}=4,5 \times 10 \times 3$ cells) were prepared from experimental crystallographic data where available (C18 ref. [38], C19 ref.[39], C20 ref. [40]). C22 was built from C20 by extending the chains and adjusting the unit cell in the $\vec{c}$ direction. It was found to be important to use large enough simulation boxes, since the melting point decreases with box size. The rationale for this is that creating a defect in too small box has an additional energetic cost because it 
Page 9 of 39 is felt via periodic boundary conditions throughout the box, whereas neighbours can adjust more easily to it in a large box. Similarly, barostat time constants of $2.5 \mathrm{ps}$ and over, comparable to the time taken by sound waves to cross the simulation box, were found necessary to ensure stability of the simulations.

Periodic boundary conditions were applied and electrostatic interactions were evaluated with the shifted potential approximation. Non-bonded interactions were cut off at $12 \AA$. All models were relaxed with the chosen force field by energy minimisation through coupling to a heat bath at $0 \mathrm{~K}$, after which a series of $\mathrm{N} \sigma \mathrm{T}$ equilibration simulations were performed at atmospheric pressure, at temperature steps of $20 \mathrm{~K}$ to just below the melting point, where smaller temperature steps of 5 or $10 \mathrm{~K}$ were adopted. Data at temperatures and pressures near the melting point were recorded every $0.5 \mathrm{ps}$ for between $200 \mathrm{ps}$ and $2 \mathrm{~ns}$. Simulations at pressures up to $1.5 \mathrm{kbar}$ were also performed. Diffusion of the chains parallel to their long axis, which leads to layer roughening, was of particular interest to us. Some of the simulations lasted over $1 \mathrm{~ns}$. It was then necesary to correcte atomic coordinates before analysis for slow drift of the simulation centre of mass. Properties of the stable phases were found to be in fair agreement with experimental data on the cell parameters, enthalpies of sublimation, melting point and pressure dependence of the melting point[32]. Here we should like to concentrate on the mechanisms of the solid-solid transformations.

\section{[Figure 8 about here.]}

Simulations of the odd alkane C19 showed a spontaneous transition to the rotator phase. Rotation of chains about their long axes was measured by a transverse vector formed by averaging the bisectors of all the $\mathrm{C}-\mathrm{C}-\mathrm{C}$ angles between two carbons chosen close to but not at the chain ends, to avoid end-chain defects. The vector thus indicates the orientation of the chain plain:

$$
\vec{T}=\sum_{i=f}^{l} \frac{1}{2}\left(\vec{r}_{i-1}-2 \vec{r}_{i}+\vec{r}_{i+1}\right),
$$

where the summation is restricted to central carbons on one side only of the chain. Figure 8 shows $90^{\circ}$ jumps of the azimuths of the normals to the carbon atom planes of two molecules in a simulation at $290 \mathrm{~K}$ in the rotator phase.

Figure 9(a) shows the variation of the cell sides in an N $\sigma \mathrm{T}$ simulation of C19 at a temperature close to the melting point of the model. Cell angles did not deviate systematically from the values in the ordered orthorhombic phase, and are not shown. Interestingly, the cell sides do not increase monotonically with temperature: $b$ passes through a maximum 
and $c$ drops, so that overall the area of the $b c$ face of the unit cell drops in the $R_{\mathrm{I}}$ phase, Page $\mathbf{1 0}$ of $\mathbf{3 9}$ in agreement with experiment $[41,42]$. The decrease of the $c$ parameter reflects end to end shortening of the chains as dihedral defects set in, mostly as kinks in the vicinity of the chain ends.

[Figure 9 about here.]

Melting of the even alkanes C18 and C20 was different. The cell sides expanded uniformly, see figure 9(b), and the cell angles varied significantly. Angle $\gamma$ dropped to $60^{\circ}$, figure 9 (c). The models in fact passed through two rotator phases before melting. We were unable to stabilize either of them, even by imposing small $(5 \mathrm{~K})$ temperature steps. Either the models were stable in their triclinic form, or they melted, going through both rotator phases at a rate increasing with the temperature. Figure 10 shows the behaviour of the ensemble of chain normals during this process.

\section{[Figure 10 about here.]}

If one compares more closely motions of individual molecules in the simulations of the odd and the even alkanes, a qualitative difference appears. Intitiation of the $R_{\mathrm{I}}$ phase of C19 is accompanied by correlated quarter turn rotational and half-chain period longitudinal jumps. Molecules remain trapped in the wrong orientation for several 10's of picoseconds. C20 behaves qualitatively differently. While molecules do make rotational jumps (see figure 10), they are short lived (about $10 \mathrm{ps)}$ ) and do not on average correspond to a lasting translation of the molecule along the chain direction. These differences are illustrated in figure 11.

[Figure 11 about here.]

These results suggest that there may be a secondary minimum on the potential energy surface of a molecule of C19 turning in its environment, responsible for the stability of the $R_{\mathrm{I}}$ phase. Absence of the minimum in the case of the even alkanes would explain why they do not readily show rotator phases. In order to check this one would like to constrain a molecule to a certain height above the surrounding layer, but relax all other degrees of freedom, the expectation being that the orientation should relax spontaneously by $90^{\circ}$ in the case of C19, but not of C20. The 'average environment' of a molecule was found by averaging the coordinates of the contents of all the unit cells in the periodic simulations at the highest temperature for which no defects were observed, and then replicating the average cell. One molecule was moved and the rest were held fixed in the averaged configuration. Only one 
Page 11 of 39 layer was considered to focus on the rotation of the mobile molecule, without interference from the planes above and below the one considered.

Constraints are normally achieved in DL_POLY by freezing atoms or by applying an isotropic restraining potential. Neither strategy is suitable here since we require freedom of movement in the plane so the chain may turn. Although DL_POLY was not designed for constraining one coordinate of a molecule, our purpose can be achieved by introducing a "condenser" of two planes of closely spaced atoms exerting a repulsive $1 / r^{6}$ energy term on one of the central carbons in the mobile molecule, sufficiently strong to constrain it to lie in the median plane of the condenser, see figure 12. The condenser is then raised by stages and the mobile molecule relaxed successive equilibrium heights by coupling to a cold heat bath.

[Figure 12 about here.]

In fact, no spontaneous rotations occurred in the above process. We therefore ran two series of minimisations, starting from the initial equilibrium height, but with the molecule either in its normal orientation or turned a quarter of a turn. Figure 13 shows the result, starting from the equilibrium orientation of the mobile molecule. Consider first C19. The potential energy rises from the initial minimum to a maximum at a shift of the centre of mass of $1.3 \AA$, corresponding to half a zig-zag of the chain and to hinderence of all the mobile methylene units with those of the first solvation shell. The potential energy then drops to a second minimum at $2.5 \AA$, with the molecule raised one repeat unit of the chain, but higher in energy because of missing interactions around the chain tail as it protrudes above the plane. During this process, the orientation of the molecule remained close to the initial value. We next turned the molecule $90^{\circ}$ before repeating the process. The turned molecule relaxes at the intial height to a secondary minimum on the global potential energy surface, about $1 \mathrm{kcal} / \mathrm{mol}$ above the ground state. But considering the vertical shift of the molecule, this minimum is actually a saddle: as the turned molecule is raised, the potential energy drops below the value on the first curve, to a minimum corresponding to the maximum on the first curve. The behaviour of the even alkanes is different, and qualitatively consistent with the progressive stability of the rotator phase in the series $\mathrm{C} 18-\mathrm{C} 22$. In $\mathrm{C} 18$, the two curves never cross, and the only minimum on the $90^{\circ}$ curve is at the crystal equilibrium position. The rotator of C18 phase was seen only recently on cooling the liquid[23]. The curves for C20 are closer, and a very slight inflexion occurs at $\mathrm{z}=1.2 \AA$ on the $90^{\circ}$ curve. In the simulations, the rotator "phases" were transient, with rotational diffusion quickly becoming isotropic, and uncorrelated with the longitudinal motion. Finally, the curves touch for C22 and that for the 
turned molecule shows a weak minimum at $\mathrm{z}=1.2 \AA$. Observation of rotator phases on heating Page $_{12}$ of 39 C20 and C22 were reported only recently[43]. It is also apparent that barriers to longitudinal diffusion in the normal crystal orientation and with increased stability of the shifted, turned molecule occur in the series $\mathrm{C} 18-\mathrm{C} 22, \mathrm{C} 19$. These features of the model correspond to increased domains of stability of the rotator phase in the real crystal. These points suggest that the $R_{\mathrm{I}}$ phases of the alkanes arise through the possibility of simultaneous translation and rotation of molecules to a secondary minimum on the potential energy surface and that it their existence and stability in the even alkanes is a quantitative question of molecular packing rather than a fundamental qualitative difference with the odd homologues.

[Figure 13 about here.]

\section{Aluminophosphate molecular sieves}

Our final example of application of DL_POLY to phase transitions is part of ongoing work on the structure and dynamics of molecular sieves doped with aromatic dye molecules. Such systems may find use as laser materials or non-linear optical components, or as bio- or environmental sensors, but the current work is stimulated by fascinating optical experiments on single molecules of oxazine dyes encapsulated in the aluminophosphate molecular sieve $\mathrm{AlPO}_{4}-5$. Fluorescence microscopy has revealed alignment of the dyes in the channels of $\mathrm{AlPO}_{4}-5$, sensitive to details of the dye structure, and in some cases rotational and absorption frequency jumps, despite the fact that the dyes must fit closely into the channels since they do not enter the already synthesized material spontaneously, but must be included during sol-gel synthesis[44]. As a first step to modelling these materials we have investigated calcined $\mathrm{AlPO}_{4}-5$ and other aluminophosphates with classical molecular dynamics simulations performed with DL_POLY and several force-fields.

Figure 14 shows the bonding in $\mathrm{AlPO}_{4}-5$. Alternating, corner sharing $\mathrm{AlO}_{4}$ and $\mathrm{PO}_{4}$ tetrahedra form 12-rings, which are in turn connected by oxygen bridges to form gasket-like sheets. Oxygen bridges along the third $(c)$ direction connect the gaskets to form an array of one-dimensional unconnected channels.

[Figure 14 about here.]

The details of the structure are still subject to debate, twenty years after the first synthesis of $\mathrm{AlPO}_{4}-5$. Wheras the usually assumed space group P6cc[45] would lead to only one Al or $\mathrm{P}$ site in the unit cell, NMR measurements have shown there may be at least three 
Page 13 of 39 different $\mathrm{Al}$ or $\mathrm{P}$ tetrahedra[46]. More than one $\mathrm{Al}$ or $\mathrm{P}$ site may exist if the space group is an orthorhombic sub-group of $P 6 c c$, as was reported by some authors on the basis of splitting of some diffraction peaks[47]. Splitting of the diffraction peaks of the parent $P 6 c c$ structure was observed by Ohnishi et al., who reported a reversible phase transition from an orthorhombic sub-group to P6cc in their samples[48]. Liu et al., on the other hand, found no evidence of splitting, but much diffuse electron diffraction attributed to excitation of rigid unit modes of the tetrahedra[49]. Assumption of $P 6 c c$ symmetry in structural refinements also leads to the conclusion that the inter-sheet oxygens are disordered, with anisotropic thermal factors, and the further difficulty that the bridge is nearly linear, wheras Al-O-P bends are usually of the order of $150^{\circ}$. It seems from these results that the framework has some degree of flexibility, and probably is quite sensitive to the synthesis, thermal history and residual water or templating agent, making it an appealing candidate for molecular dynamics simulations.

We performed simulations on models of $2 \times 2 \times 3$ cells in the $a, b$ and $c$ directions, for both the hexagonal and the orthorhombic starting configurations. Unconstrained minimisations and simulated annealing were performed by coupling to a cold heat bath in DL_POLY. Constant temperature and stress simulations were performed with the Berendsen thermoand barostats[5]. Ewald summation was applied to evaluate the long range forces. Short range terms were cut off at $12 \AA$. The time step was $0.5 \mathrm{fs}$. Three empirical force fields were used:(a) one with formal charges on all atoms, polarisable oxygen atoms and 6-exp functions to represent the covalent part of the interaction, derived by Gale and Henson (GH)[50]; (b) the second with partial charges and a Morse potential energy function, due to Kitao et al. (MS-Q)[51]; (c) the model with partial charges and 6-exp interactions, by van Beest et al.[52] . Fluctuating charges in the MS-Q model in response to changes in the instantaneous bonding of atoms were replaced by average values found in calculations with the force field on another aluminophosphate sieve, VPI-5[51] $\left(q_{\mathrm{Al}}=1.4078, q_{\mathrm{P}}=0.5678\right)$.

Naturally, the minimised or averaged dynamical structures differ slightly from the some of the experimental data. As an aid to identification the structures, diagrams of the projections on the $a b$ plane of all the $\mathrm{Al}$ (or P) tetrahedra in the simulation were prepared. The unit cell of $\mathrm{AlPO}_{4}-5$ contains two 12-rings superposed along the $c$ axis. Figure 15 shows schematically the all the Al tetrahedra of the unit cell viewed down the $c$ axis for the ideal case of a true 6 -fold axis at the centre of the 12-ring. If the $\mathrm{Al}$ atoms of the tetrahedra are all centred on the origin and the oxygen atoms are projected onto the $a b$ plane, we expect a single spot at $x=0, y=0$, and 12 spots lying on a circle. Tilt of the tetrahedra, or differences between 
them in actual simulations cause the spots to split up into clusters of points. Examination ${ }_{\text {Page }} \mathbf{1 4}$ of 39 of such diagrams for the number of spots and the presence of symmetries such as reflections or rotations is helpful to determine the number of distinct tetrahedra and the space group.

[Figure 15 about here.]

Summarising our findings, which will be developped elsewhere[4], iso-stress (1 bar) unconstrainsed energy minimisation of the model with the GH force field led to a structure in good overall agreement with the experimental data. On inspection, we find the space group Pnn2 with final energy $-268.0700 \mathrm{eV}$ per $\mathrm{AlPO}_{4}$ unit. The inter-sheet oxygen bridge angles range from $157^{\circ}$ to $180^{\circ}$. Unconstrained minimisation during simulated annealing converged to structures at first sight hexagonal $(b / a=\sqrt{ } 3 \pm .005)$. But closer examination revealed over 10 orthorhombic minima within $.005 \mathrm{eV}$ per $\mathrm{AlPO}_{4}$ unit of the lowest structure (Pnn2 above), such as a $P c c 2$ structure with a doubling of the unit cell in the $a$ and $b$ directions $\left(a=2 \times 13.80 \AA, b=2 \times 23.97 \AA, c=8.41 \AA, \alpha=\beta=\gamma=90 \pm .001^{\circ},-268.0642 \mathrm{eV}\right.$ per $\mathrm{AlPO}_{4}$ unit) and 24 distinct tetrahedra. The main differences between these minima are in different disorders of the inter-ssheet oxygens bridges.

[Figure 16 about here.]

Simulated annealing with both the BKS and the MS-Q models yielded a single, triclinic structure, albeit very close to hexagonal, in three settings at roughly $120^{\circ}$ from one another, aligned along the line of centres of adjacent 12-rings. Figure 16 compares the overall lowest structures obtained with the GH and the MS-Q models. Interestingly, successive gaskets in the MS-Q model are offset in the $a b$ plane, while all the inter-sheet oxygens are aligned along $c$, similar to what was found by earlier authors for the BKS model (and reproduced in our work)[53].

Figure 17 compares the Al tetrahedra of the GH and the MS-Q models at $300 \mathrm{~K}$. Three tetrahedra can be distinguished in the MS-Q model, analagous to the findings of Peeters et al.[46] from NMR data, but only one in the GH model. Average P6cc symmetry, with only one tetrahedron, was observed in all simulations with the G-H model, even at temperatures as low as $50 \mathrm{~K}$ (c.f. the $c a .0 .005 \approx 40 \mathrm{~cm}^{-1}$ between the minima above).

[Figure 17 about here.]

The MS-Q model of $\mathrm{AlPO}_{4}-5$ actually undergoes an order-disorder phase transition to $P 6 c c$ at just above room temperature, as was revealed by projection of the tetrahedra and http://mc.manuscriptcentral.com/tandf/jenmol 
Page 15 of 39 by following the temperature dependence of the ratio $b / a$ of the unit cell. At low enough temperatures the model is locked in one or other of the three degenerate minima. On heating to room temperature, jumps of the whole model to the adjacent minima are observed, with excursions of the ratio $b / a$ from its initial value, figure 18(a), while at high temperatures the ratio converges to $\sqrt{ } 3$, the value expected for hexagonal symmetry, figure $18(\mathrm{~b})$. This behaviour parallels the reversible phase transition reported by Ohnishi et al.[48].

[Figure 18 about here.]

In summary, the partial charge models MS-Q and BKS give similar results, with some features in good agreement with experimental data, such as a phase transition to hexagonal symmetry at around room temperature and the presence of several tetrahedra in the low temperature or ordered phase, but with less satisfactory agreement with experiment on other points, such as the offset of successive sheets and the linearity of the Al-O-P intersheet bridges. The Gale-Henson model applied to this system has exactly complementary good and bad points. Successful simulations of other aluminophosphate sieves with the MS$\mathrm{Q}$ force field, including the first order transition from $\mathrm{AlPO}_{4}-53(\mathrm{~B})$ to $\mathrm{AlPO}_{4}-53(\mathrm{C})$ with recoordination of some atoms (where the Gale-Henson model does not succeed), nonetheless lead us to think that there may be some underlying similarity between the simulations of $\mathrm{AlPO}_{4}-5$ with partial charge models and reality. It is to be hoped that the structure will be determined at low temperatures, or that ab initio energy minimisations will shed light on this curious material.

\section{Conclusion}

The present examples of order-disorder transitions show just a few of the many capabilities of DL_POLY as a versatile tool for simulation of solids. Future developments will no doubt benefit from the new domain decomposition parallelisation scheme in DL_POLY_3, which will allow study of larger models. Whereas studies of the equilibrium properties of different phases can be done with current models, study of bigger models and longer runs are most important for direct simulation of systems close to a phase transition, in order to alleviate the effects of finite size and critical slowing down. 
Part of the simulations were performed on the parallel computers of the M3PEC regional consortium for high performance computing, Université de Bordeaux I.

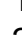

2

4

5

6

7

8

9

10

11

12

13

14

15

16

17

18

19

20

21

22

23

24

25

26

27

28

29

30

31

32

33

34

35

36

37

38

39

40

41

42

43

44

45

46

47

48

49

50

51

52

53

54

55

56

57

58

59

60 
[1] M. Causà, R. Dovesi, C. Pisani, and C. Roetti, Phys. Rev. B 33, 1308 (1986).

[2] M.-P. Habas, R. Dovesi, and A. Lichanot, J. Phys: Condens. Matter 10, 6897 (1998).

[3] I. P. Swainson and M. T. Dove, J. Phys: Condens. Matter 7, 1771 (1995).

[4] P. Bordat and R. Brown, (2006), to be published.

[5] H. J. C. Berendsen, J. P. M. Potsma, W. F. van Gunsteren, A. DiNola, and J. R. Haak, J. Chem. Phys. 81, 3684 (1984).

[6] A. Almenningen, O. Bastiansen, L. Fernholt, B. N. Cyvin, S. J. Cyvin, and S. Samdal, J. Mol. Struct. 128, 59 (1985).

[7] P. Bordat and R. Brown, Chem. Phys. 246, 323 (1999).

[8] J. L. Baudour, Y. Délugeard, and H. Cailleau, Acta. Cryst. B32, 150 (1976).

[9] H. Cailleau and A. Dworkin, Mol. Cryst. Liq. Cryst. 50, 217 (1979).

[10] R. E. Lechner, B. Toudic, and H. Cailleau, J. Phys. C: Solid State Phys. 17, 405 (1984).

[11] B. Toudic and R. E. Lechner, J. Phys. C: Solid State Phys. 17, 5503 (1984).

[12] B. Toudic, H. Cailleau, J. Galliear, and R. E. Lechner, J. Phys. I France 2, 829 (1992).

[13] K. Yokoi and T. Nishikawa, Mol. Phys. 90, 705 (1997).

[14] P. Bordat and R. Brown, Chem. Phys. Lett. 291, 153 (1998).

[15] P. Bordat and R. Brown, J. Chem. Phys. 116, 229 (2002).

[16] D. Wolf, P. Keblinskii, S. R. Phillpot, and J. Eggebrecht, J. Chem. Phys. 110, 8254 (1999).

[17] J. L. Baudour, Acta. Cryst. B47, 935 (1991).

[18] J. L. Baudour and H. Cailleau, Acta. Cryst. B33, 1773 (1977).

[19] C. de la Riva, C. Glowatz, C. Kryschi, M.-H. Lemée-Cailleau, H. Cailleau, M. Bertault, and J. Even, Chem. Phys. Lett. 277, 132 (1997).

[20] R. Sayle and E. J. Milner-White, Trends in Biochemical Sciences 20, 374 (1995). 
[21] M.-H. Lemé-Cailleau and R. Brown, (2006), to be published.

[22] P. D. Reilly and J. L. Skinner, J. Chem. Phys. 102, 1540 (1995).

[23] E. B. Sirota and A. B. Herhold, Polymer 41, 8781 (2002).

[24] F. Guillaume, J.-P. Ryckaert, V. Rodriguez, L. Gonzalez Mac Dowell, P. Girard, and A. Dianoux, Phase Transitions 76, 823 (2003).

[25] T. Gowrek, R. Zaleski, and J. Wawryszczuk, Chem. Phys. Lett. 394, 90 (2004).

[26] M. Maroncelli, S. P. Qi, H. L. Strauss, and R. G. Snyder, J. Am. Chem. Soc. 104, 6237 (1982).

[27] Y. Kim, H. L. Strauss, and R. G. Snyder, J. Phys. Chem. 93, 7520 (1989).

[28] B. Ewen, E. W. Fischer, W. Piesczek, and G. Strobl, J. Chem. Phys. 61, 5265 (1974).

[29] H. Yamakawa, S. Masukawa, H. Kurosu, S. Kuroki, and I. Ando, J. Chem. Phys. 111, 7110 (1999).

[30] P. Espeau, L. Roblès, D. Mondieig, Y. Haget, M. A. Cuevas-Diarte, and H. A. J. Oonk, J. Chim. Phys. 93, 1217 (1996).

[31] A. Würflinger, D. Mondieig, F. Rajabalee, and M. Cuevas-Diarte, Z. Naturforsch. A 56, 1 (2001).

[32] A. Marbeuf and R. Brown, J. Chem. Phys. 124, 054901 (2006).

[33] J. P. Ryckaert, M. Klein, and I. R. McDonald, Mol. Phys. 83, 439 (1994).

[34] J. P. Ryckaert, Physica A 213, 50 (1995).

[35] L. Gonzalez Mac Dowell, F. Guillaume, J. P. Ryckaert, P. Girard, V. Rodriguez, and A.-J. Dianoux, Physica B 234-236, 106 (1997).

[36] H. Sun, J. Phys. Chem. B 102, 7338 (1998).

[37] W. Smith and T. R. Forester, DL_POLY 2.13 Manual, CCLRC, Daresbury Laboratory, Daresbury, Nr. Warrington WA4 4AD, UK, 1996.

[38] S. C. Nyburg and H. Lüth, Acta Cryst. B28, 2992 (1992).

[39] P. Négrier, private communication (unpublished). 
${ }_{39}[40]$ S. C. Nyburg and A. R. Gerson, Acta Cryst. B48, 103 (1992).

[41] E. B. Sirota, D. M. Singer, and H. E. King Jr., J. Chem. Phys. 100, 1542 (1994).

[42] E. B. Sirota and J. H. E. King, Science 281, 143a (1998).

[43] S. Chazhengina, E. N. Kotelnikova, I. V. Filippova, and S. K. Filatov, J. Mol. Struct. 647, 243 (2002).

[44] C. Seebacher, C. Hellriegel, C. BrÃd'uchle, M. Ganschow, and D. WÃúhrle, J. Phys. Chem. B 107, 5445 (2003).

[45] J. M. Bennett, J. P. Cohen, E. M. Flanigen, J. J.Pluth, and J. V. Smith, ACS Symp. Ser. (PUBLISHER, ADDRESS, 1983), Vol. 218, p. 109.

[46] M. P. J. Peeters, L. J. M. van de Ven, J. W. de Haan, and J. H. C. van Hooff, J. Phys. Chem. 97, 8254 (1993).

[47] A. J. Mora, A. N. Fitch, M. Cole, R. Goyal, R. H. Jones, H. Jobic, and S. W. Carr, J. Mater. Chem. 6, 1831 (1996).

[48] N. Ohnishi, S. Qiu, O. Terasaki, T. Kajitani, and K. Hiraga, Microporous Mater. 2, 73 (1993).

[49] Y. Liu, R. L. Withers, and L. LorÃln, Microporous Mesoporous Mater. 42, 103 (2001).

[50] J. D. Gale and N. Henson, J. Chem. Soc. Faraday Trans. 90, 3175 (1994).

[51] O. Kitao, E. Demiralp, T. Cagin, S. Dasgupta, M. Mikami, K. Tanabe, and W. A. Goddard III, Comp. Mater. Sci. 14, 135 (1999).

[52] B. W. H. van Beest, G. J. Kramer, and R. A. van Santen, Phys. Rev. Lett. 64, 1955 (1990).

[53] A. de Man, W. Jacobs, J. Gilson, and R. van Santen, Zeolites 12, 826 (1992).

[54] T. Ikeda, K. Miyazawa, F. Izumi, Q. Huang, and A. Santoro, J. Phys. Chem. solids 60, 1531 (199).

[55] M.-H. Lemée-Cailleau, Ph.D. thesis, Université de Rennes I, . 
1 (a) $p$-terphenyl, showing the torsions $\phi_{1}$ and $\phi_{2}$ active in the order-disorder transition; (b) Schematic molecular packing in the $a, b$ plane, viewed down the $c$ axis (long molecular axis), showing the room temperature unit cell (dotted), the low temperature cell (solid) and the conventional pseudo-monoclinic cell (dashed). Inner(black) and outer(shaded) rings appear end on. Reproduced from ref. [7]. . . . . . . . . . . . . . . . . . .

2 (a): Scatter diagram of the instantaneous inter-ring torsions, $\phi_{1}$ and $\phi_{2}$ at $300 \mathrm{~K}$. The dashed line has a slope of -1 ; ((b): Joint distribution of the instantaneous twists out of mean plane of an outer $\left(\theta_{1}\right)$ and the central $\left(\theta_{2}\right)$ ring. The dashed line has a slope of $-1 / 2 ;\left((c)\right.$ : Simulated distribution of $\theta_{2}$ at $180 \mathrm{~K}$ (histogramme) compared with the analysis of neutron diffraction data at $200 \mathrm{~K}$ by Baudour et al.[18] ( bold smooth curve); ((d): Same for room temperature; ((e): Effective potentials for twisting the central ring out of the mean plane (bold $180 \mathrm{~K}$, thin $300 \mathrm{~K}$ ) deduced from the distributions in ((c) and $((d)$.Reproduced from ref. [7]. . . . . . . . . . . . . . .

3 Simulated temperature dependence of the inter-phenyl torsion angles at (a) 1 bar, (b) $2.5 \mathrm{kbar}$; (c) Pressure dependence at $150 \mathrm{~K}$; (d) Part of the experimental phase diagram of $p$-terphenyl, showing the order-disorder transition temperature as a function of pressure (Raman scattering data redrawn from ref. [55]) and the simulated points in parts $(a)-(c) \ldots . . . . .$.

4 Schematic of (a) ferroelastic and (b) antiphase walls in the $b c$ plane of $p$ terphenyl. Central rings shown grey (red), outer rings black (blue). Molecules at the interface have indeterminate ring torsions or frustrated interactions with one or other of their neighbours in the $a$ direction, no matter which ring orientation that is adopted to optimise packing against the opposite neighbour. 25

5 Model of an anti-phase domain wall in $p$-terphenyl (18x8x4 molecules). The antiphases are coloured grey and brown respectively. The external sheets coloured blue are frozen. . . . . . . . . . . . . . . . .

6 Development of the domain wall set up in figure 5, viewed normal to the $a b$ plane after $80 \mathrm{ps}$ of dynamics at $50 \mathrm{~K}$. Molecules in each of the four planes of the model are shown separately for clarity. The initial position of the domain wall is shown by the black line in the first plane on the left. Molecules coloured blue are frozen in the simulation, while those coloured green are in the normal conformation for their initital domain. Molecules with ring inversion compared to their initial domain are coloured red. Grey molecules are flat (torsions under $6^{\circ}$ compared to the ideal values $15-27^{\circ}$ in the low

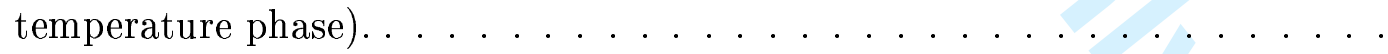

7 Top row: Molecular packing of (a) the even (C18) and (b) the odd (C19) alkanes; $(c)-(d)$ detail of the packing within a layer. . . . . . . . . . .

8 Sample tracks of the azimuths in the $a b$ plane of normals to the carbon chains of two molecules of C19 in a simulation at $290 \mathrm{~K}$, showing $90^{\circ}$ jumps between the preferred orientations in the $R_{\mathrm{I}}$ phase. . . . . . . . . . . .

9 (a) Simulated variation of the cell parameters of $\mathrm{C} 19$ at $290 \mathrm{~K}$, in the vicinity of the $O \rightarrow R_{I}$ transition. Thin line: $a$; medium: $b$; thick: $c$; (b) Cell sides of $\mathrm{C} 20$ at $290 \mathrm{~K}$ (thin line), $310 \mathrm{~K}$ (medium) and $320 \mathrm{~K}$ (thick); (c) Cell angles of $\mathrm{C} 20$ at the same temperatures as in (b). Reproduced from ref. [32]. . . 
10 (a) Scatter diagram of the instantaneous azimuths of the short molecular axes during the first part of the simulation of C20 at $320 \mathrm{~K}$ in figure 9 (b-c). Note the transition of the preferred orientations at $t=150 \mathrm{ps}$ (arrow); (b) Sliding average distributions of the orientations. Reproduced from ref. [32]. . . . . .

11 (a)Sample tracks of the azimuth of the chain normal and the height of the centre of mass above the layer plane, of a molecule of C19 during initiation of the $R_{\mathrm{I}}$ phase at $285 \mathrm{~K}$; (b)Same for a molecule of $\mathrm{C} 20$ at $300 \mathrm{~K}$ during transition throught the unstable rotator phases. Note the change in timescale of the orientational excursion. . . . . . . . . . . . . . . .

12 "Setup" to constrain the height of the centre of mass of a molecule while relaxing all other degrees of freedom: atoms in the plates of the "condenser" exert repulsive interactions on one atom (ball) of the molecule (sticks), constraining it to lie in the median plane of the condenser. . . . . . . . . . . .

13 Total potential energy vs. height of the molecular center of mass on withdrawing one molecule from its plane, in its natural orientation (thin line) or turned $90^{\circ}$ (thick).Reproduced from ref. [32]. . . . . . . . . . . . . . .

14 (a) Perspective view down the $c$ axis of the contents of the unit cell of $\mathrm{AlPO}_{4}$ 5 (P6cc space group, data from Ikeda et al.[54]), showing two 12-rings with alternating $\mathrm{AlO}_{4}$ and $\mathrm{PO}_{4}$ tetrahedra: $\mathrm{Al}$ green (medium grey), $\mathrm{P}$ yellow (light grey), O red (dark grey); (b) orthographic projection of structure onto the $(\mathrm{a}, \mathrm{b})$ plane $(2 \times 2 \times 2$ unit cells), showing sheets of 12-rings; (c) orthographic view along the $(\mathbf{a}+\mathbf{b}) / 2$ direction, showing inter-sheet Al-O-P bridges. . . .

15 Schematic view of the projection onto the $a b$ plane of the oxygen atoms of the centred $\mathrm{Al}$ tetrahedra of both rings of the unit cell of $\mathrm{AlPO}_{4^{-}}-5$ assuming an ideal six-fold axis along $c . \ldots \ldots \ldots$

16 Projections onto the $a b$ plane of (a) The experimental Pnn2 structure of Ikeda et al. [54]; (b) The lowest minimim of the Gale-Henson model; (c) one setting of the three-fold degenerate minimum of the MS-Q model; (d)-(f) Corresponding

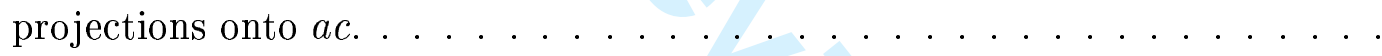

17 (a) Scatter diagram of the projected $\mathrm{O}$ atoms of $\mathrm{Al}$ tetrahedra in the $\mathrm{G}-\mathrm{H}$ model at $300 \mathrm{~K}$; (b) Same for the MS-Q model. Only one kind of tetrahedron is present on average in the GH model, whereas three can be distinguished in the MS-Q model, related to the 3 kinds of $\mathrm{Al}$ in the Pnn2 structure of calcined $\mathrm{AlPO}_{4}-5$ in ref. [54]. . . . . . . . . . . . . . .

18 (a) Time dependence at different temperatures of the ratio $b / a$ for $\mathrm{N} \sigma \mathrm{T}$ simulations of $\mathrm{AlPO}_{4}-5$ with the MS-Q model. The dashed lines shows the value $\sqrt{ } 3$ correponding to hexagonal symmetry. Successive curves vertically offset by 0.05 for clarity. ; (b) Time averaged distribution of the ratio at the same temperatures, showing convergence of the ratio to $\sqrt{ } 3$ (arrow). Vertical offset

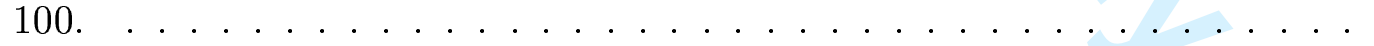




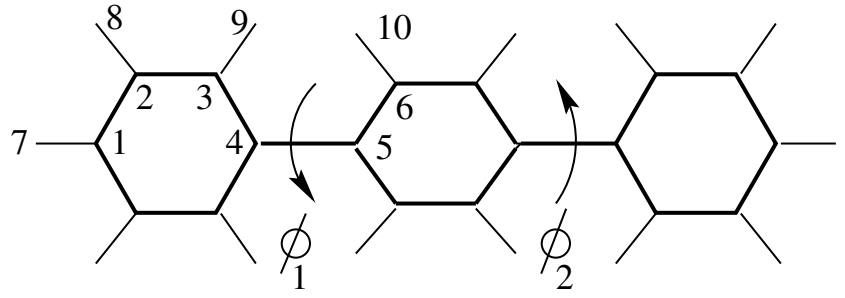

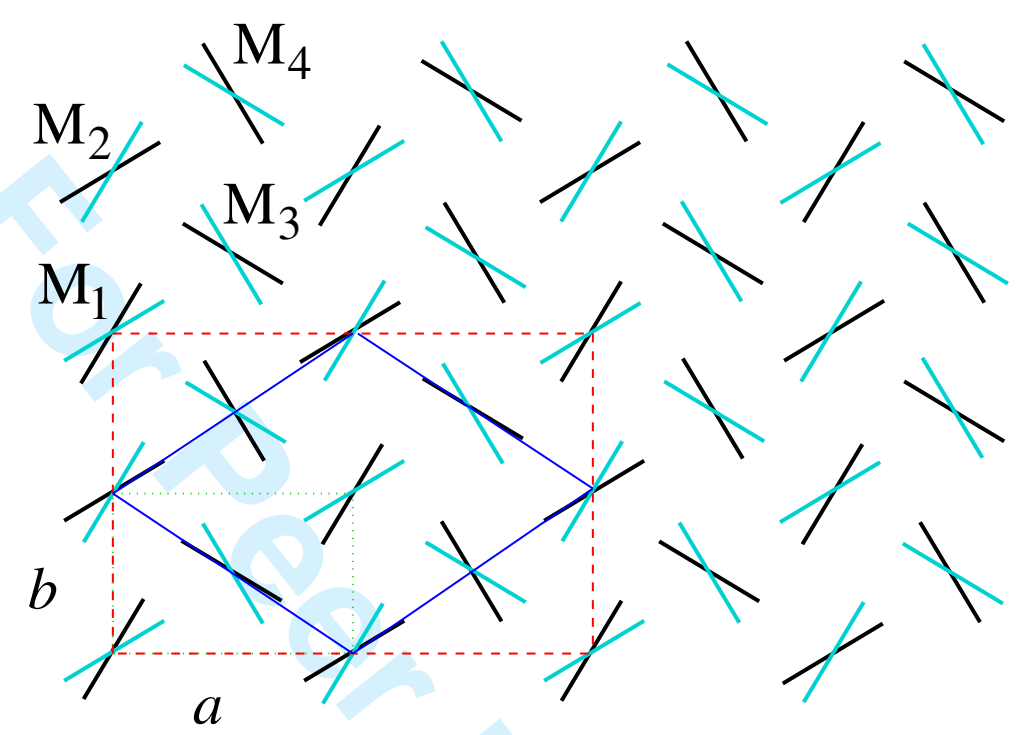

(b)

Figure 1: (a) p-terphenyl, showing the torsions $\phi_{1}$ and $\phi_{2}$ active in the order-disorder transition; (b) Schematic molecular packing in the $a, b$ plane, viewed down the $c$ axis (long molecular axis), showing the room temperature unit cell (dotted), the low temperature cell (solid) and the conventional pseudo-monoclinic cell (dashed). Inner(black) and outer(shaded) rings appear end on. Reproduced from ref. [7]. 

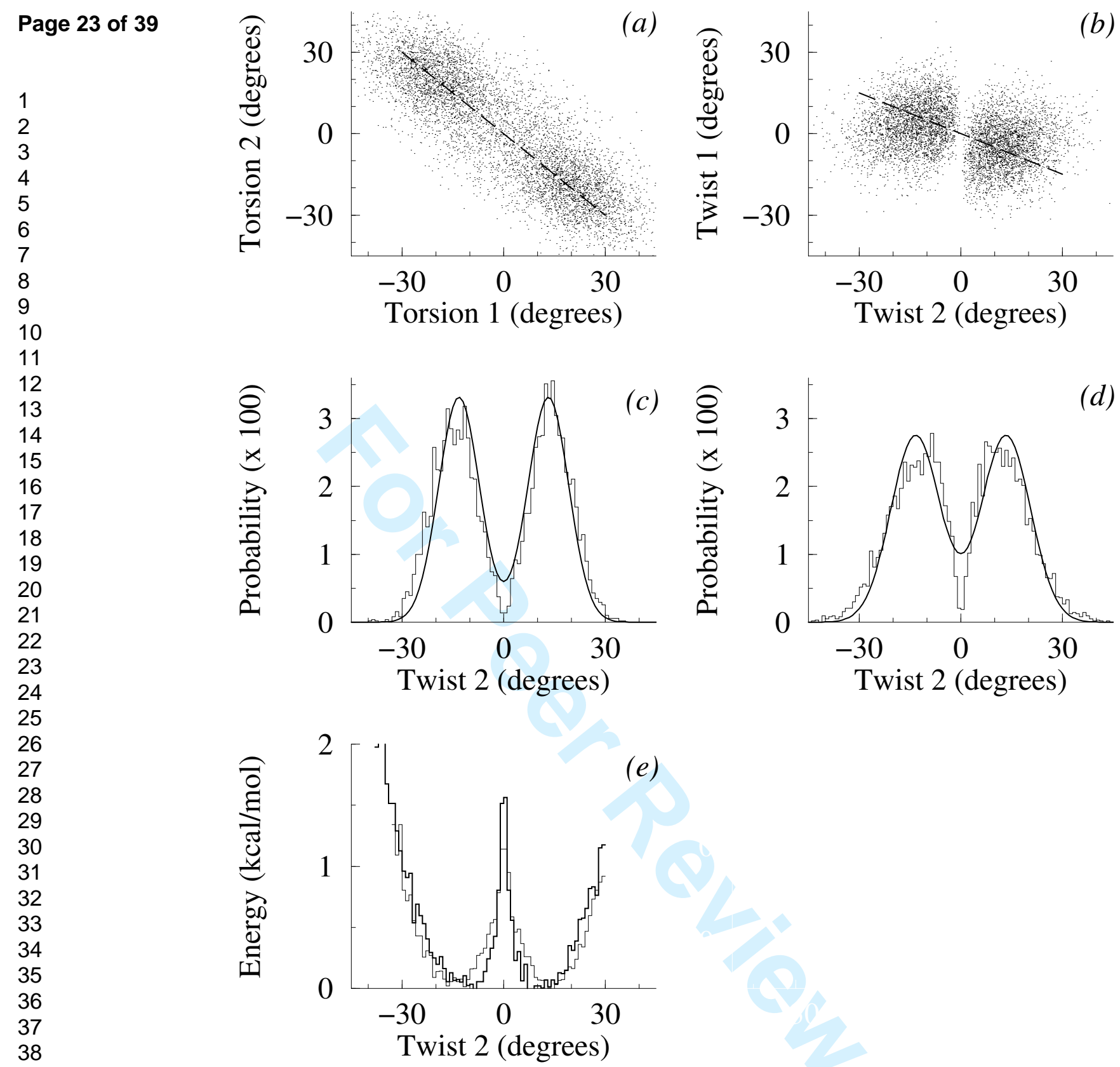

Figure 2: (a): Scatter diagram of the instantaneous inter-ring torsions, $\phi_{1}$ and $\phi_{2}$ at $300 \mathrm{~K}$. The dashed line has a slope of $-1 ;((b)$ : Joint distribution of the instantaneous twists out of mean plane of an outer $\left(\theta_{1}\right)$ and the central $\left(\theta_{2}\right)$ ring. The dashed line has a slope of $-1 / 2$; ( $c$ ): Simulated distribution of $\theta_{2}$ at $180 \mathrm{~K}$ (histogramme) compared with the analysis of neutron diffraction data at $200 \mathrm{~K}$ by Baudour et al.[18] ( bold smooth curve); ((d): Same for room temperature; ((e): Effective potentials for twisting the central ring out of the mean plane (bold $180 \mathrm{~K}$, thin $300 \mathrm{~K})$ deduced from the distributions in $((c)$ and $((d)$.Reproduced from ref. [7]. 
(a)

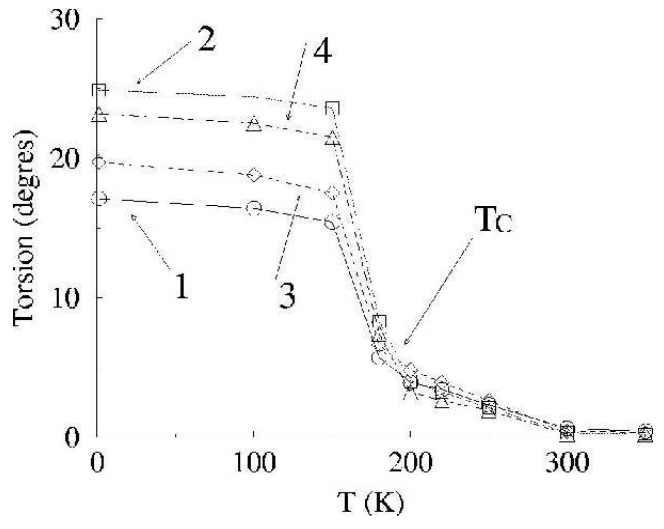

(c)

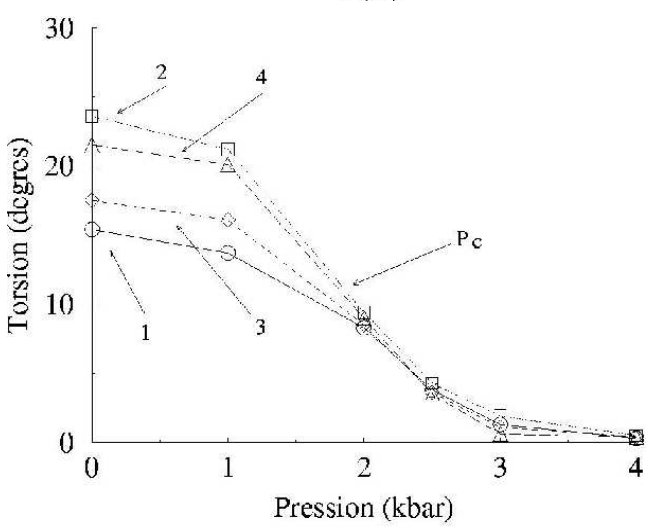

(b)

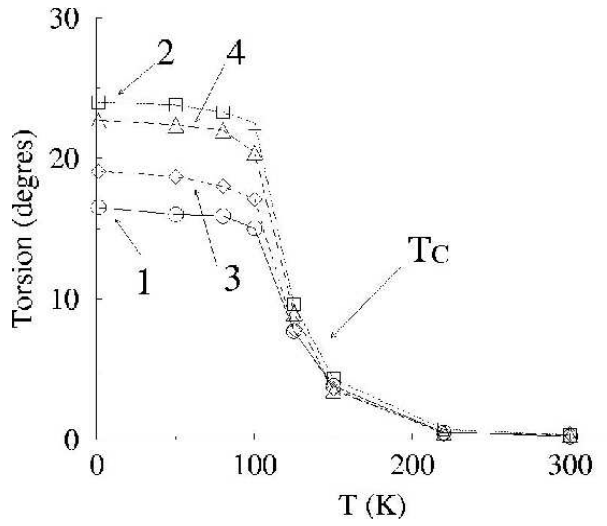

(d)

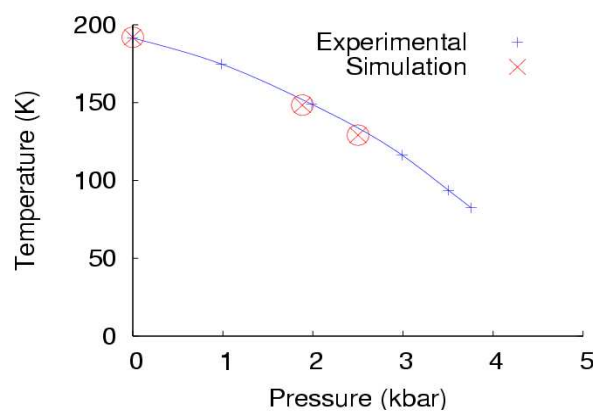

Figure 3: Simulated temperature dependence of the inter-phenyl torsion angles at (a) 1 bar, (b) $2.5 \mathrm{kbar}$; (c) Pressure dependence at $150 \mathrm{~K}$; (d) Part of the experimental phase diagram of $p$-terphenyl, showing the order-disorder transition temperature as a function of pressure (Raman scattering data redrawn from ref. [55]) and the simulated points in parts (a)-(c) 
(a)
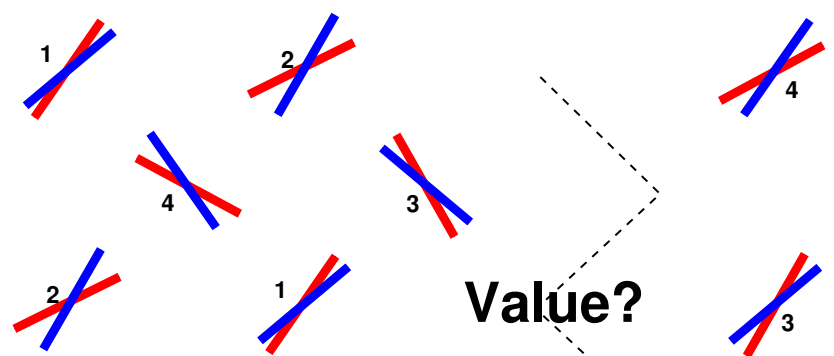

13

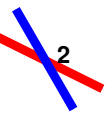

Valúe?
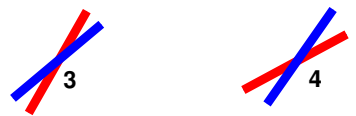

3
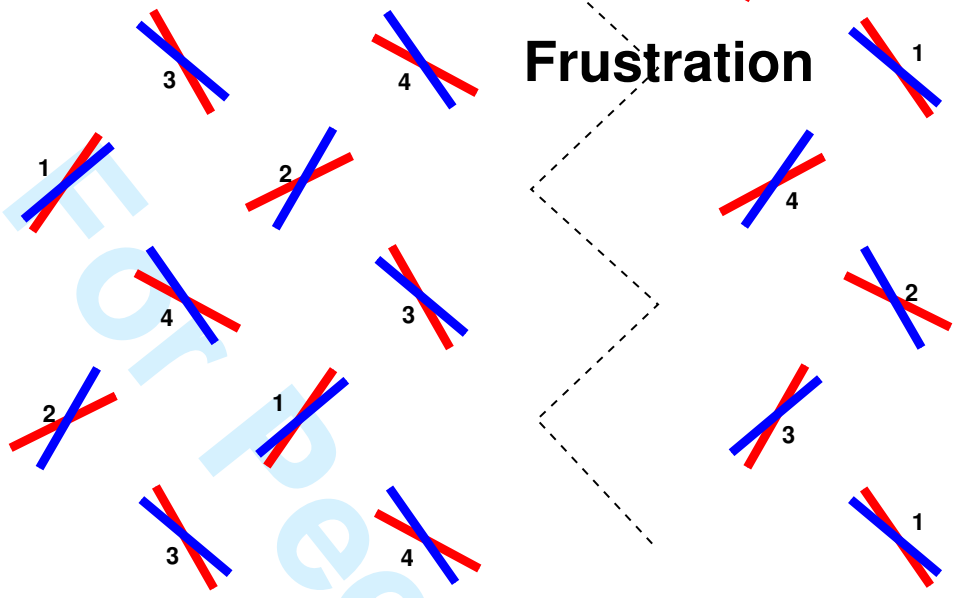

Domain growth
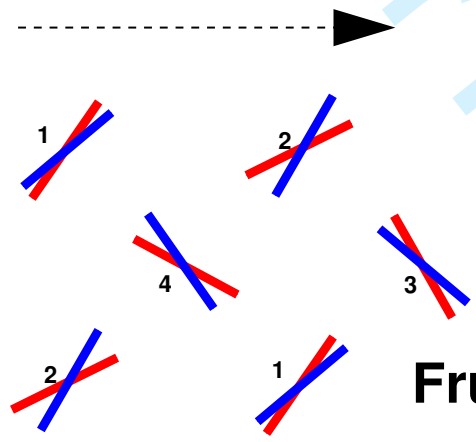

Frustráation
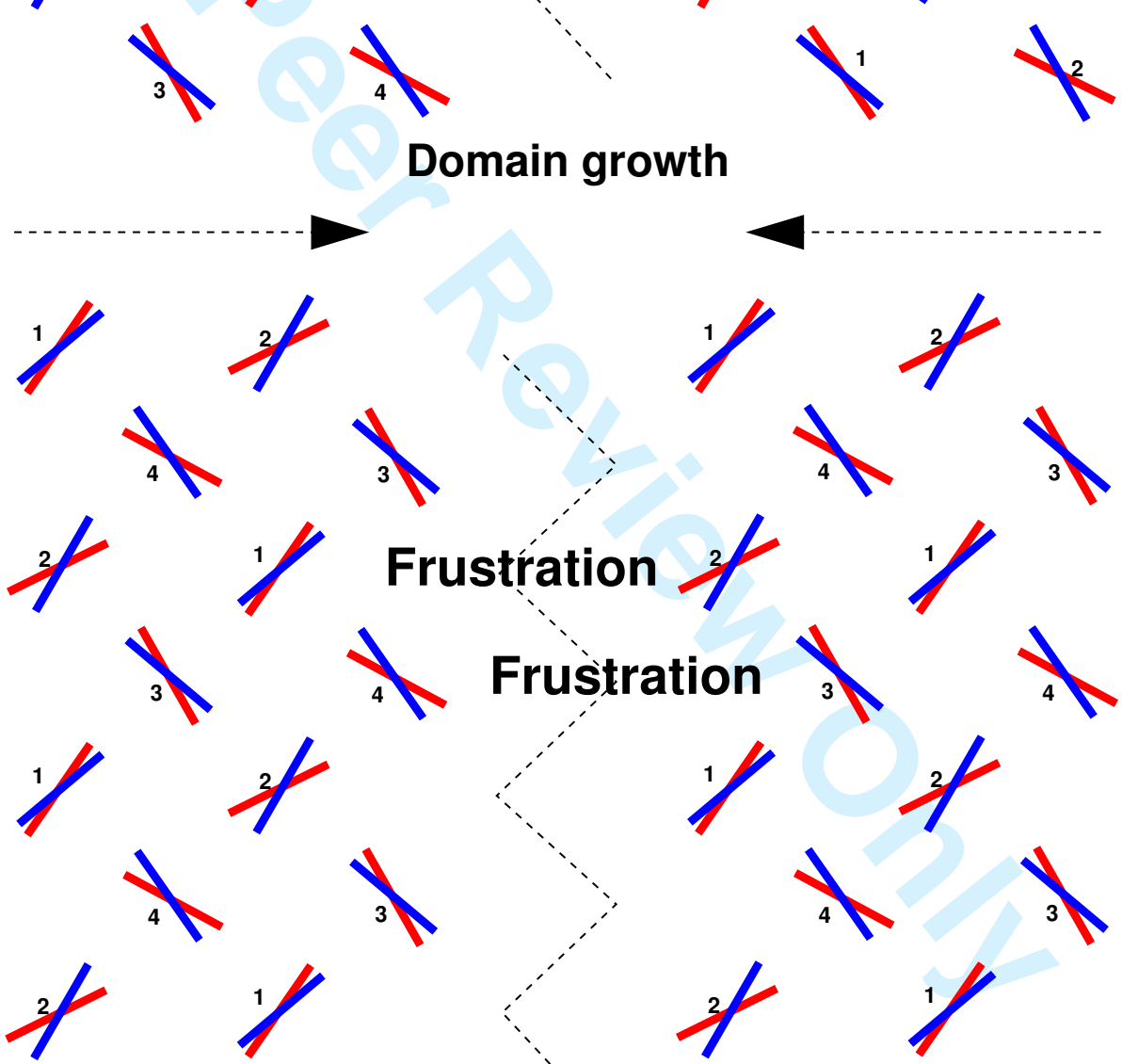

(b)
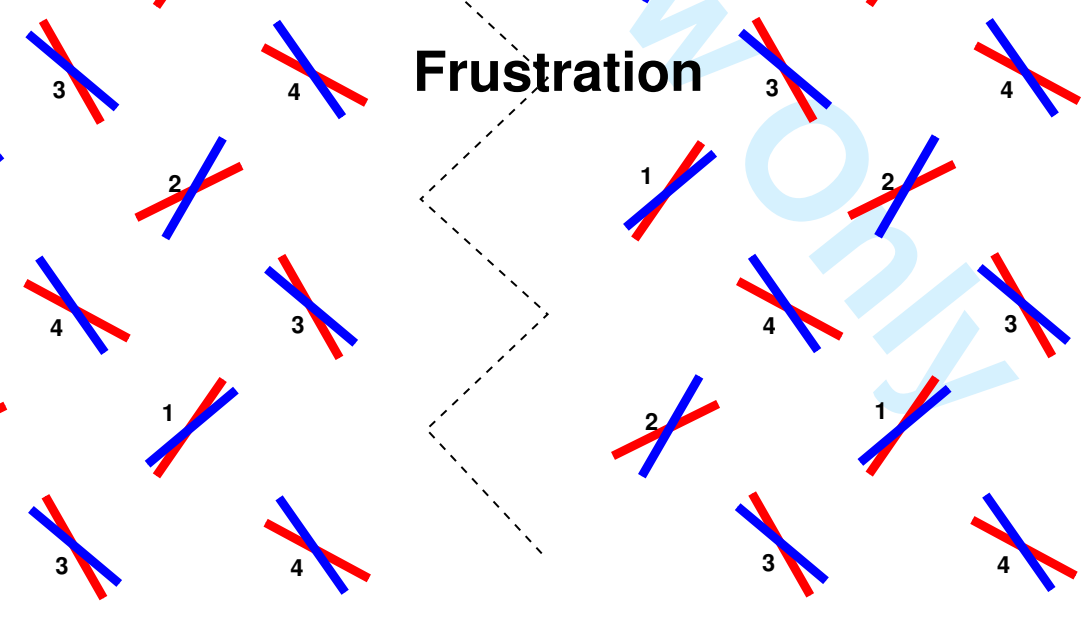

Figure 4: Schematic of (a) ferroelastic and (b) antiphase walls in the bc plane of $p$-terphenyl. Central rings shown grey (red), outer rings black (blue). Molecules at the interface have indeterminate ring torsions or frustrated interactions with one or other of their neighbours in the $a$ direction, no matter which ring orientation is adopted to optimise packing against the opposite neighbour. 


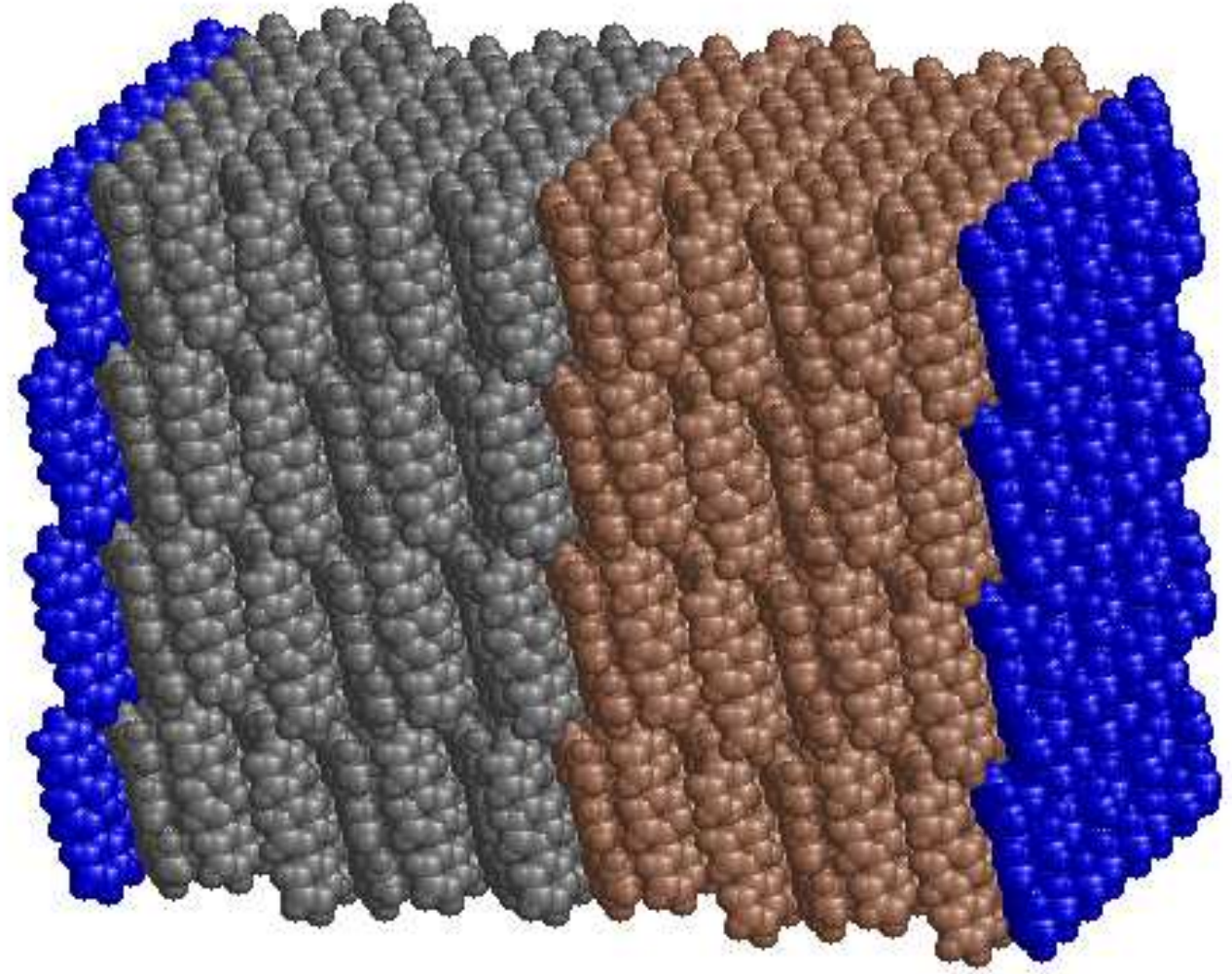

Figure 5: Model of an anti-phase domain wall in $p$-terphenyl (18x8 44 molecules). The antiphases are coloured grey and brown respectively. The external sheets coloured blue are frozen. 


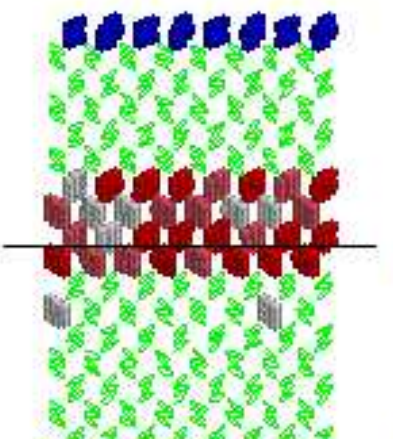

werenters

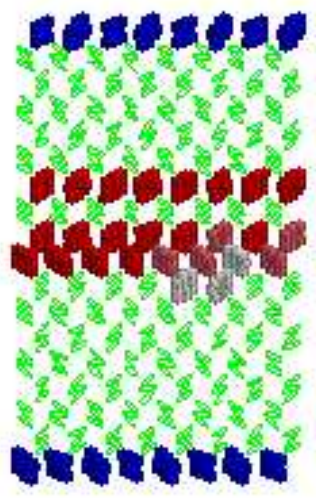

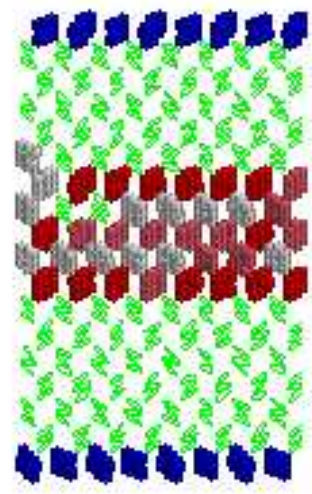

Figure 6: Development of the domain wall set up in figure 5, viewed normal to the $a b$ plane after 80 ps of dynamics at $50 \mathrm{~K}$. Molecules in each of the four planes of the model are shown separately for clarity. The initial position of the domain wall is shown by the black line in the first plane on the left. Molecules coloured blue are frozen in the simulation, while those coloured green are in the normal conformation for their initital domain. Molecules with ring inversion compared to their initial domain are coloured red. Grey molecules are flat (torsions under $6^{\circ}$ compared to the ideal values $15-27^{\circ}$ in the low temperature phase). 
(a)
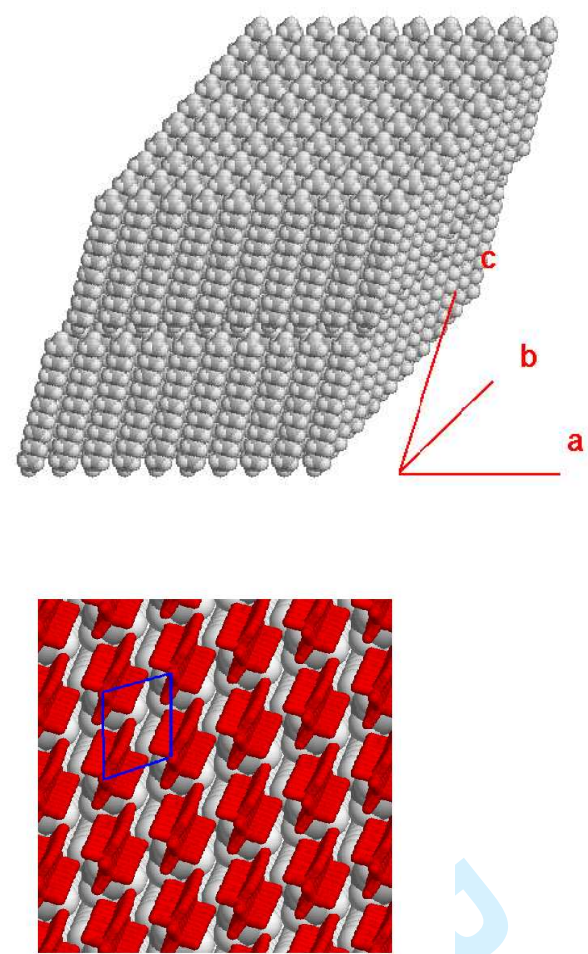

(c)

Figure 7: Top row: Molecular packing of (a) the even (C18) and (b) the odd (C19) alkanes; (c)- (d) detail of the packing within a layer. (b)

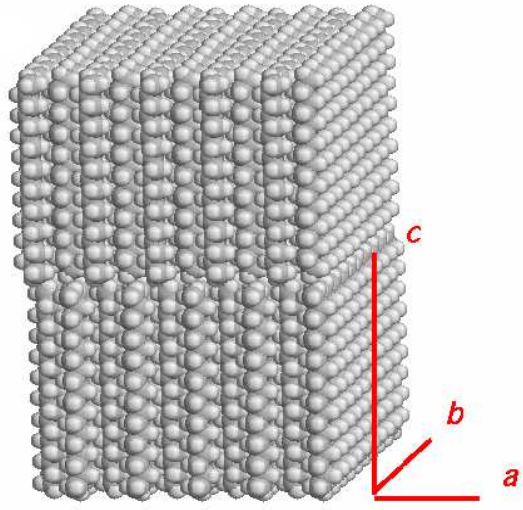

Page 28 of 39 


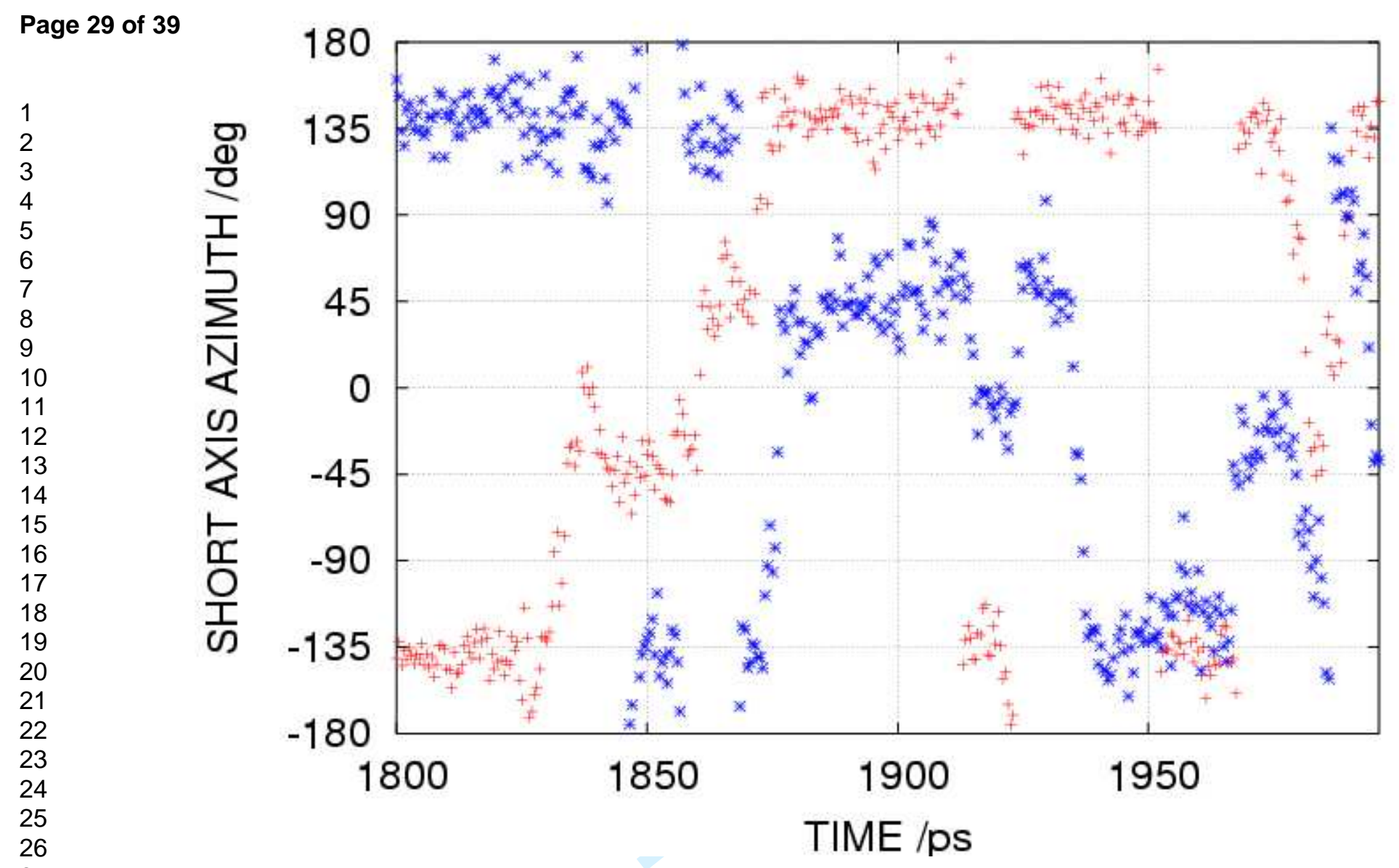

Figure 8: Sample tracks of the azimuths in the $a b$ plane of normals to the carbon chains of two molecules of $\mathrm{C} 19$ in a simulation at $290 \mathrm{~K}$, showing $90^{\circ}$ jumps between the preferred orientations in the $R_{\mathrm{I}}$ phase. 

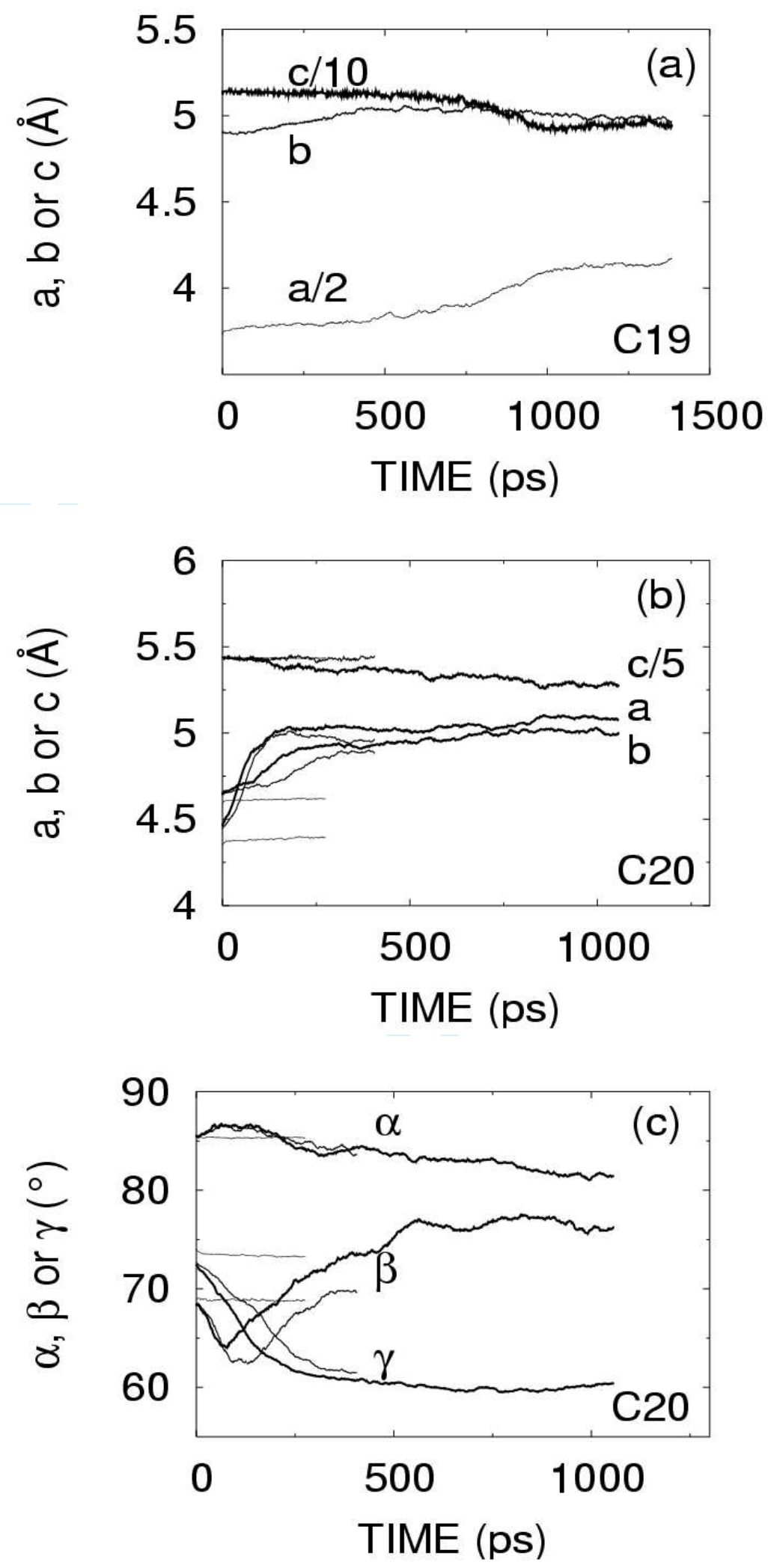

Figure 9: (a) Simulated variation of the cell parameters of C19 at $290 \mathrm{~K}$, in the vicinity of the $O \rightarrow R_{I}$ transition. Thin line: $a$; medium: $b$; thick: $c$; (b) Cell sides of $\mathrm{C} 20$ at $290 \mathrm{~K}$ (thin line), $310 \mathrm{~K}$ (medium) and $320 \mathrm{~K}$ (thick); (c) Cell angles of C20 at the same temperatures as in (b). Reproduced from ref. [32]. 

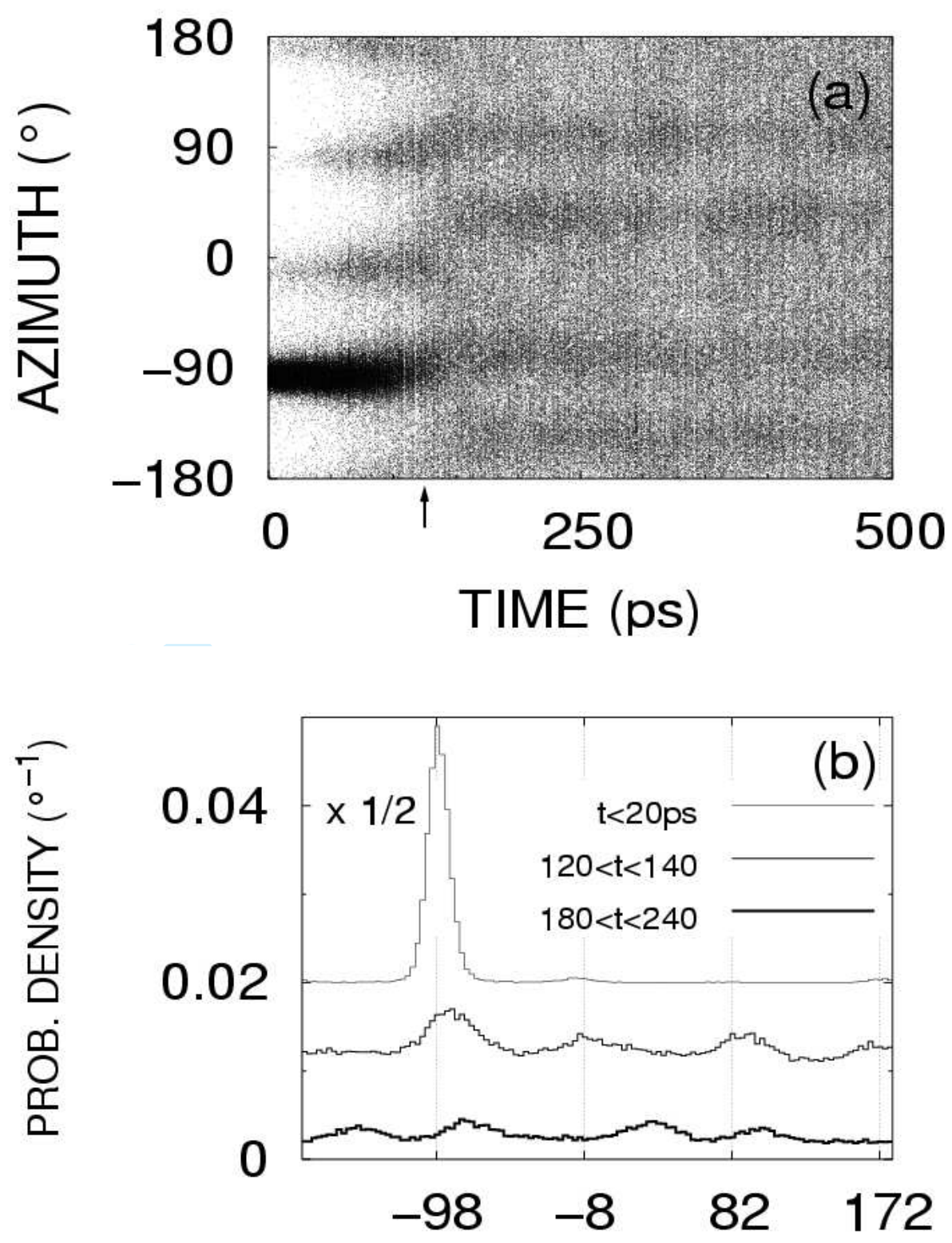

AZIMUTH $\left({ }^{\circ}\right)$

Figure 10: (a) Scatter diagram of the instantaneous azimuths of the short molecular axes during the first part of the simulation of $\mathrm{C} 20$ at $320 \mathrm{~K}$ in figure 9 (b-c). Note the transition of the preferred orientations at $t=150 \mathrm{ps}$ (arrow); (b) Sliding average distributions of the orientations. Reproduced from ref. [32]. 


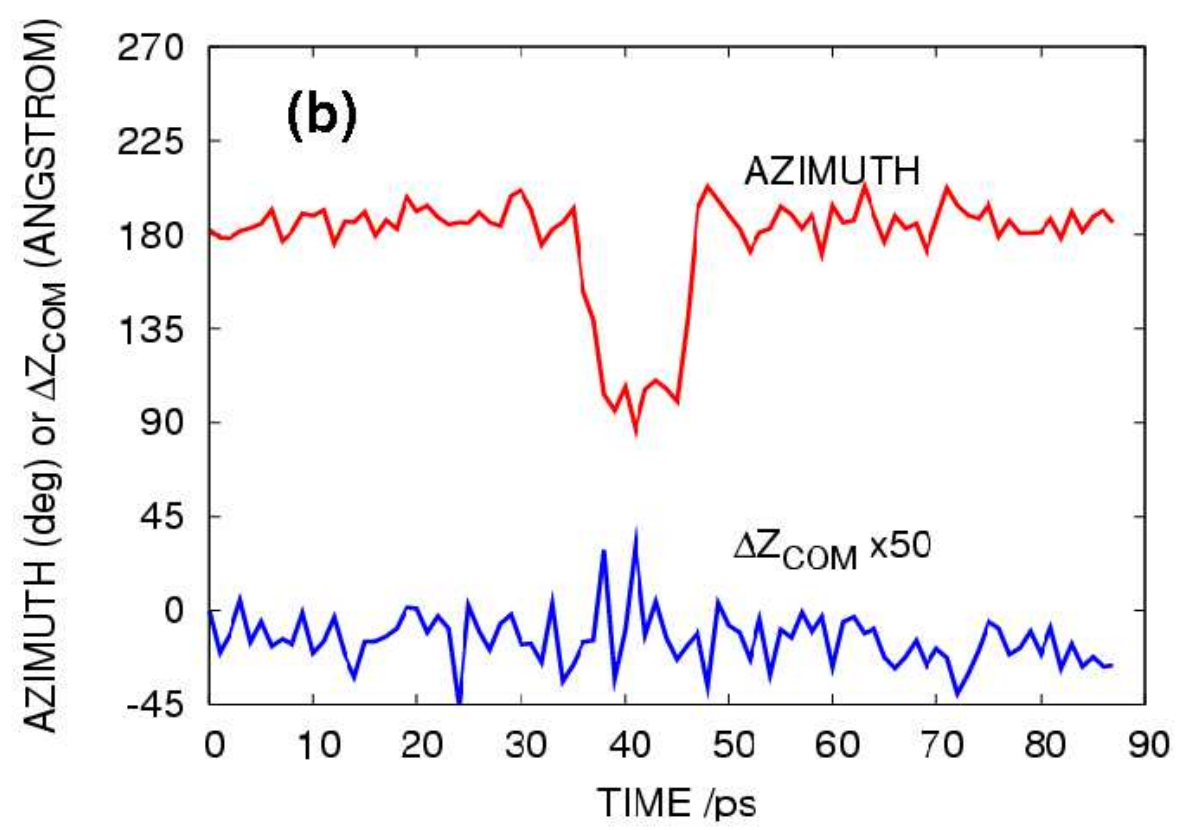

Figure 11: (a)Sample tracks of the azimuth of the chain normal and the height of the centre of mass above the layer plane, of a molecule of C19 during initiation of the $R_{\mathrm{I}}$ phase at $285 \mathrm{~K}$ ; (b)Same for a molecule of $\mathrm{C} 20$ at $300 \mathrm{~K}$ during transition throught the unstable rotator phases. Note the change in timescale of the orientational excursion. 
Figure 12: "Setup" to constrain the height of the centre of mass of a molecule while relaxing all other degrees of freedom: atoms in the plates of the "condenser" exert repulsive interactions on one atom (ball) of the molecule (sticks), constraining it to lie in the median plane of the condenser. 


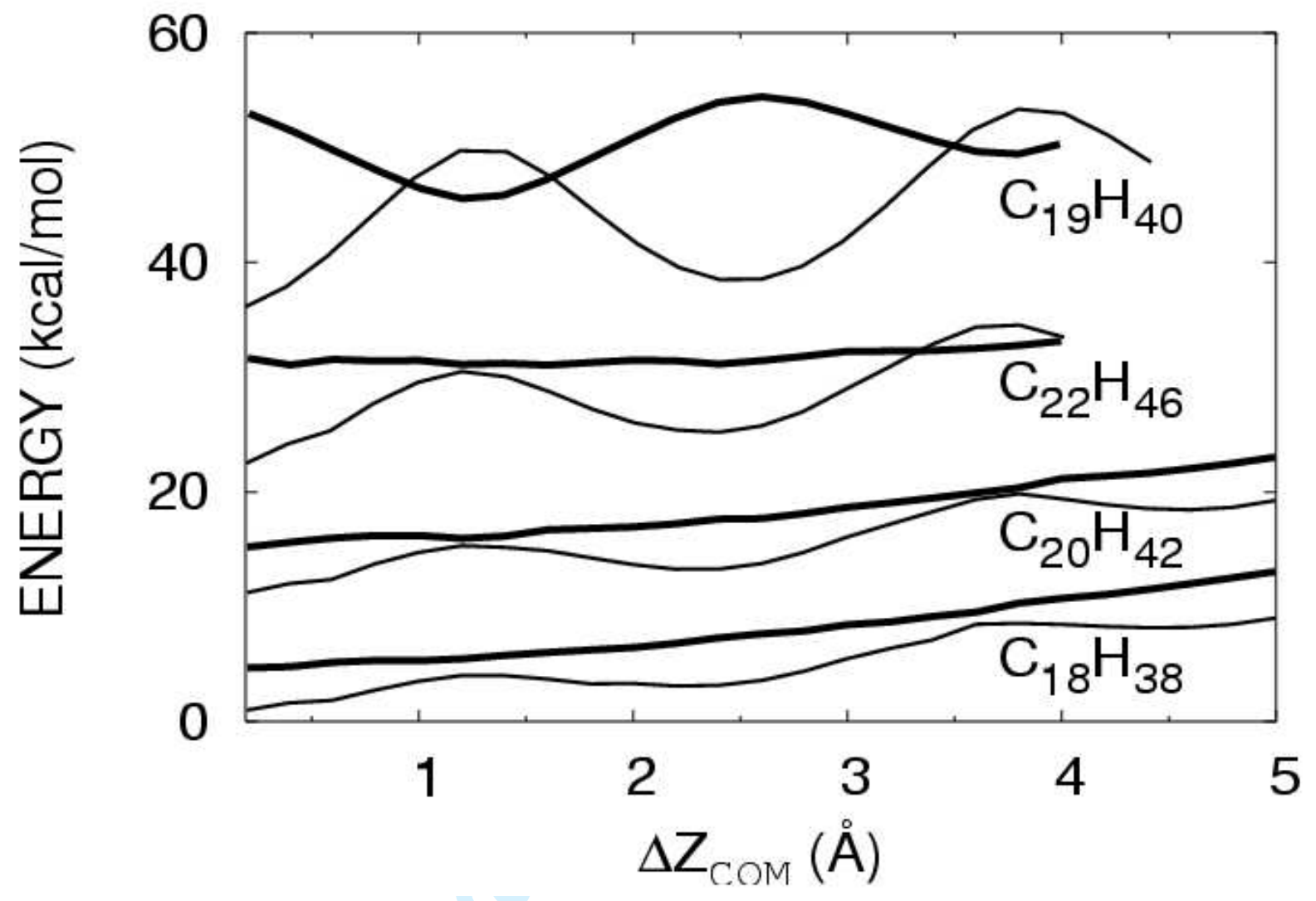

Page 34 of 39

Figure 13: Total potential energy vs. height of the molecular center of mass on withdrawing one molecule from its plane, in its natural orientation (thin line) or turned $90^{\circ}$ (thick).Reproduced from ref. [32]. 


\section{Page 35 of 39}

(a)

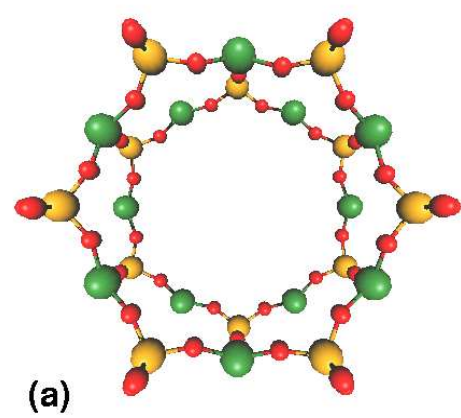

(b)
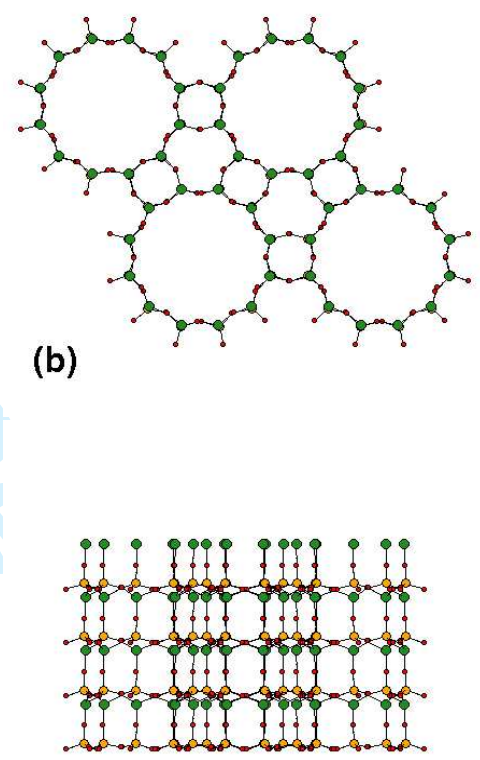

(c)

Figure 14: (a) Perspective view down the $c$ axis of the contents of the unit cell of $\mathrm{AlPO}_{4^{-}} 5$ (P6cc space group, data from Ikeda et al.[54]), showing two 12-rings with alternating $\mathrm{AlO}_{4}$ and $\mathrm{PO}_{4}$ tetrahedra: $\mathrm{Al}$ green (medium grey), $\mathrm{P}$ yellow (light grey), $\mathrm{O}$ red (dark grey); (b) orthographic projection of structure onto the $(\mathrm{a}, \mathrm{b})$ plane $(2 \times 2 \times 2$ unit cells), showing sheets of 12-rings; (c) orthographic view along the $(\mathbf{a}+\mathbf{b}) / 2$ direction, showing inter-sheet Al-O-P bridges. 
1

2

3

4

5

8

9

10

11

12

13

14

15

16

17

18

19

20

21

22

23

24

25

26

27

28

29

30

31

32

33

34

35

36

37

38

39

40

41

42

43

44

45

46

47

48

49

50

51

52

53

54

55

56

57

58

59

60

Figure 15: Schematic view of the projection onto the $a b$ plane of the oxygen atoms of the centred $\mathrm{Al}$ tetrahedra of both rings of the unit cell of $\mathrm{AlPO}_{4}-5$ assuming an ideal six-fold axis along $c$. 


Pag


1
2
2
3
4
5
5
6
7
8
9
9
10
11
12
12
13
14
15
16
16
17
18
19
20
21
2

$a$

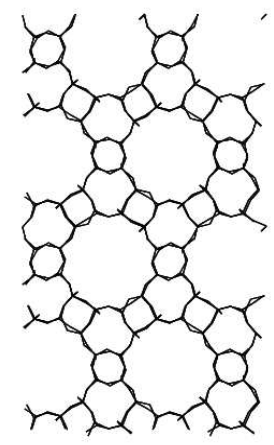

$b$

$c$
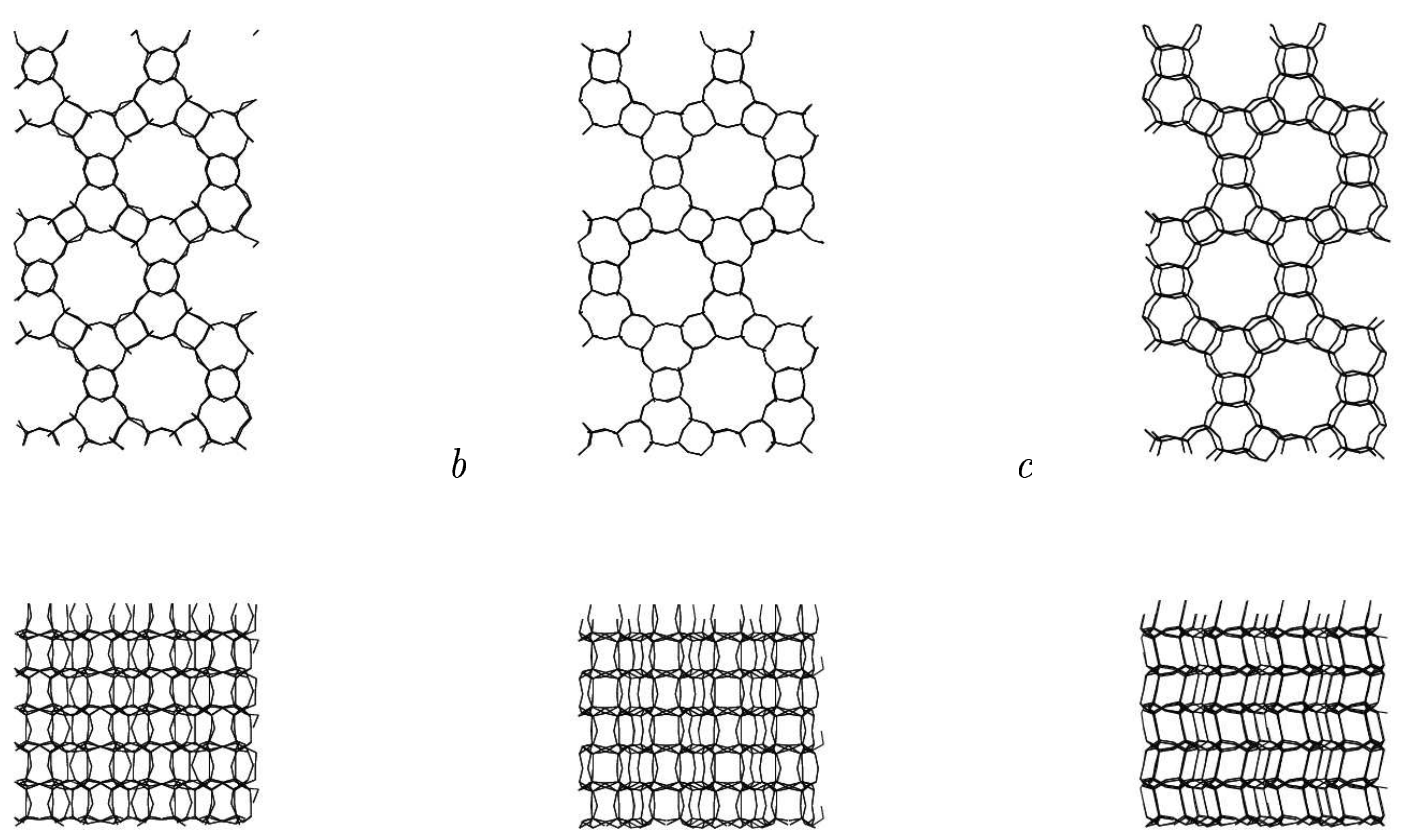

$d$

$e$

$f$

Figure 16: Projections onto the $a b$ plane of (a) The experimental Pnn2 structure of Ikeda $e t$ al. [54]; (b) The lowest minimim of the Gale-Henson model; (c) one setting of the three-fold degenerate minimum of the MS-Q model; $(d)-(f)$ Corresponding projections onto ac. 
Figure 18: (a) Time dependence at different temperatures of the ratio $b / a$ for $\mathrm{N} \sigma \mathrm{T}$ simulations of $\mathrm{AlPO}_{4}-5$ with the MS-Q model. The dashed lines shows the value $\sqrt{ } 3$ correponding to hexagonal symmetry. Successive curves vertically offset by 0.05 for clarity. ; (b) Time averaged distribution of the ratio at the same temperatures, showing convergence of the ratio to $\sqrt{ } 3$ (arrow). Vertical offset 100. 